\title{
Control Theory Meets POMDPs: A Hybrid Systems Approach
}

\author{
Mohamadreza Ahmadi, Nils Jansen, Bo Wu, and Ufuk Topcu
}

\begin{abstract}
Partially observable Markov decision processes (POMDPs) provide a modeling framework for a variety of sequential decision making under uncertainty scenarios in artificial intelligence (AI). Since the states are not directly observable in a POMDP, decision making has to be performed based on the output of a Bayesian filter (continuous beliefs); hence, making POMDPs intractable to solve and analyze. To overcome the complexity challenge of POMDPs, we apply techniques from control theory. Our contributions are fourfold: (i) We begin by casting the problem of analyzing a POMDP into analyzing the behavior of a discrete-time switched system. Then, (ii) in order to estimate the reachable belief space of a POMDP, i.e., the set of all possible evolutions given an initial belief distribution over the states and a set of actions and observations, we find over-approximations in terms of sub-level sets of Lyapunovlike functions. Furthermore, (iii) in order to verify safety and performance requirements of a given POMDP, we formulate a barrier certificate theorem, wherein we show that if there exists a barrier certificate satisfying a set of inequalities along with the belief update equation of the POMDP, the safety and performance properties are guaranteed to hold. In both cases (ii) and (iii), the calculations can be decomposed and solved in parallel. (iv) We show that the conditions we formulate can be computationally implemented as a set of sum-of-squares programs. We illustrate the applicability of our method by addressing two problems in active ad scheduling and machine teaching.
\end{abstract}

\section{INTRODUCTION}

A formal model for planning subject to stochastic behavior is a Markov decision process (MDP) [1], where an agent chooses to perform an action under full knowledge of the environment it is operating in. The outcome of an action is a probability distribution over the system states. Many applications, however, allow only partial observability of the current system state, say via noisy sensor outputs [2], [3], [4]. Partially observable Markov decision processes (POMDPs) extend MDPs to account for such partial information [5]. Upon certain observations, the agent infers the likelihood of the system being in a certain state, called the belief state. The belief state together with an update function form a (typically uncountably infinite) MDP, referred to as the belief MDP [6], [7], [8]. However, solving a POMDP exactly, i.e., synthesizing

This work was supported by AFOSR FA9550-19-1-0005, AFRL FA9550-19-1-0169, DARPA D19AP00004, NSF 1646522, NWO OCENW.KLEIN.187, and NSF 1652113 grants.

M. Ahmadi is with the Center for Autonomous Systems and Technologies at the California Institute of Technology, 1200 E. Calif. Blvd., MC 10444, Pasadena, CA 91125. e-mail: (\{mrahmadi\}@caltech.edu). B. Wu and $\mathrm{U}$. Topcu are with the Oden Institute for Computational Engineering and Sciences, University of Texas, Austin, $201 \mathrm{E} 24 \mathrm{th}$ St, Austin, TX 78712, USA e-mail: ( $\{$ bwu3, utopcu $\} @$ utexas.edu). N. Jansen is with the Department of Software Science (SWS) at the Radboud University, Institute for Computing and Information Sciences, PO Box 9010, 6500 GL Nijmegen, Netherlands. e-mail: (\{n.jansen\}@science.ru.nl). a policy ensuring optimality or a certain performance, is carried out by assessing the entire belief MDP, rendering the problem undecidable [7], [9]. Several promising approximate point-based methods via finite abstraction of the belief space are proposed in the literature [10], [11], [12], [13]. At the heart of these methods is the observation that, given an initial belief $b_{0}$ (the initial probability of the system being in some state), the set of possible evolutions emanating from $b_{0}$ under arbitrary action and observation sequences, called the reachable belief space is much smaller than the whole belief space [14]. Hence, only a finite number of samples or points from the reachable belief space can be considered for decision making.

Nonetheless, computing the reachable belief space is nontrivial and fundamental in analyzing the convergence of approximate POMDP solutions. In fact, Pineu et. al. [15] showed that point-based value iteration can approximate the solution of the POMDP sufficiently close, whenever the total reward function is a discounted sum of the rewards over time, the samples are sufficiently close to each other, and if the sampling is carried out over all of the reachable belief space. In another vein, Lee et. al. [16] demonstrated that an approximate POMDP solution can be computed in time polynomial in the covering number (the number of balls covering a set) of the reachable belief space. Yet, the problem lies in the fact that the reachable belief space is not known a priori and therefore it is hard to make judgements on the convergence rate of a given approximate method. Several techniques have been proposed for estimating the reachable belief space mainly based on heuristics [17], [18], "smarter" sampling methods, such as importance sampling [19], and graph search [20], [21]. However, these methods are difficult to adapt from one problem setting to another and are often ad hoc.

Even with a reachable belief space at hand, the approximate techniques do not provide a guarantee for safety or performance. That is, it is not clear whether the probability of satisfying a pre-specified safety/ performance requirement is an upper-bound or a lower-bound for a given POMDP. Establishing guaranteed safety and performance is of fundamental importance in safety-critical applications, e.g. aircraft collision avoidance [22] and Mars rovers [23].

In this paper, we use notions from control theory to analyze a POMDP without the need to solve it explicitly. In this sense, our method follows the footsteps of Lyapunov's second method for verifying the stability of all solutions of an ODE without the need to solve it or the application of barrier certificate theorems for safety verification without the need to solve an ODE for all initial conditions in a given set. Our main contributions are fourfold:

(i) We propose a switched system representation for the 
belief evolutions of POMDPs;

(ii) We present a method based on Lyapunov-like functions to over-approximate the reachable belief space, thus allowing us to verify reachability objectives. These overapproximations are in terms of sub-level sets of the latter functions. We demonstrate how these calculations can be decomposed in terms of the POMDP actions;

(iii) We propose a method based on barrier certificates to verify safety and performance of a given POMDP. We show how the calculations of the barrier certificates can be decomposed in terms of actions;

(iv) We formulate a set of sum-of-squares programs for finding the Lyapunov functions and barrier certificates to verify safety and performance and over-approximate the reachable belief space, respectively.

We illustrate the efficacy of our methodology with two examples from cyber-physical systems and artificial intelligence.

Preliminary results related to the formulation of the barrier certificates for POMDPs applied to privacy verification and robust decision making were disseminated in [24], [25].

The rest of the paper is organized as follows. In the next section, we briefly describe the POMDP formalism. In Section III, we present the problems studied in this paper. In Section IV, we formulate a hybrid system representation for belief MDP. In Section V, we show how we can use Lyapunovlike functions to approximate the reachable belief space. In Section VI, we propose barrier certificate theorem for safety/ performance verification of POMDPs. In Section VII, we propose a set of sum-of-squares programs for computational implementation of the proposed results. In Section VIII, we elucidate the proposed methodology with examples. Finally, in Section IX, we conclude the paper and give directions for future research.

Notation: $\mathbb{R}_{>0}$ denotes the set $[0, \infty) . \mathbb{Z}$ denotes the set of integers and $\mathbb{Z}_{\geq c}$ for $c \in \mathbb{Z}$ implies the set $\{c, c+1, c+2, \ldots\}$. $\mathcal{R}[x]$ accounts for the set of polynomial functions with real coefficients in $x \in \mathbb{R}^{n}, p: \mathbb{R}^{n} \rightarrow \mathbb{R}$ and $\Sigma \subset \mathcal{R}$ is the subset of polynomials with a sum-of-squares decomposition; i.e, $p \in \Sigma[x]$ if and only if there are $p_{i} \in \mathcal{R}[x], i \in\{1, \ldots, k\}$ such that $p=p_{i}^{2}+\cdots+p_{k}^{2}$. For a finite set $A,|A|$ denotes the number of elements in $A$ and $\operatorname{co}\{A\}$ denotes the convex hull of $A$. In particular, for a family of indexed functions $f_{a}$ : $A \rightarrow Z, a \in A, \operatorname{co}\left\{f_{a}\right\}_{a \in A}$ denotes the convex combination of $f_{a}, a \in A$, i.e., $c o\left\{f_{a}\right\}_{a \in A}=\sum_{a \in A} \beta_{a} f_{a}$ where $0 \leq \beta_{a} \leq 1$ and $\sum_{a \in A} \beta_{a}=1$.

\section{Partially Observable Markov Decision PROCESSES}

A POMDP is a sequential decision-making modeling framework, which considers not only the stochastic outcomes of actions, but also the imperfect state observations [26].

Definition 1. A POMDP $\mathcal{P}$ is a tuple $\left(Q, p_{0}, A, T, Z, O\right)$, where

- $Q$ is a finite set of states with indices $\{1,2, \ldots, n\}$;

- $p_{0}: Q \rightarrow[0,1]$ defines the distribution of the initial states, i.e., $p_{0}(q)$ denotes the probability of starting at $q \in Q$;
- A is a finite set of actions;

- $T: Q \times A \times Q \rightarrow[0,1]$ is the transition probability, where

$$
\begin{array}{r}
T\left(q, a, q^{\prime}\right):=P\left(q_{t}=q^{\prime} \mid q_{t-1}=q, a_{t-1}=a\right), \\
\forall t \in \mathbb{Z}_{\geq 1}, q, q^{\prime} \in Q, a \in A .
\end{array}
$$

- $Z$ is the set of all possible observations. Often, $z \in Z$ is an incomplete projection of the world state $q$, contaminated by noise;

- $O: Q \times A \times Z \rightarrow[0,1]$ is the observation probability (sensor model), where

$$
\begin{aligned}
O(q, a, z):=P\left(z_{t}=z \mid q_{t}=q, a_{t-1}=a\right) & \\
& \forall t \in \mathbb{Z}_{\geq 1}, q \in Q, a \in A, z \in Z .
\end{aligned}
$$

Since the states are not directly accessible in a POMDP, decision making requires the history of observations. Therefore, we need to define the notion of a belief $b$ (or the posterior) as sufficient statistics for the history [27]. The belief in $q$ at time $t, b_{t}(q)$, is the probability of being in state $q$ at time $t$ given that action $a_{t-1}$ was taken at time $t-1$ and observation $z_{t}$ is received [2]. Given a POMDP, the belief at $t=0$ is defined as $b_{0}(q)=p_{0}(q)$ and $b_{t}(q)$ can be obtained by a Bayesian filter as

$$
\begin{aligned}
b_{t}\left(q^{\prime}\right) & =P\left(q^{\prime} \mid z_{t}, a_{t-1}, b_{t-1}(q)\right) \\
& =\frac{P\left(z_{t} \mid q^{\prime}, a_{t-1}, b_{t-1}(q)\right) P\left(q^{\prime} \mid a_{t-1}, b_{t-1}(q)\right)}{P\left(z_{t} \mid a_{t-1}, b_{t-1}(q)\right)} \\
& =\frac{P\left(z_{t} \mid q^{\prime}, a_{t-1}, b_{t-1}(q)\right)}{P\left(z_{t} \mid a_{t-1}, b_{t-1}(q)\right)} \\
& \times \sum_{q \in Q} P\left(q^{\prime} \mid a_{t-1}, b_{t-1}(q), q\right) P\left(q \mid a_{t-1}, b_{t-1}(q)\right) \\
& =\frac{O\left(q^{\prime}, a_{t-1}, z_{t}\right) \sum_{q \in Q} T\left(q, a_{t-1}, q^{\prime}\right) b_{t-1}(q)}{\sum_{q^{\prime} \in Q} O\left(q^{\prime}, a_{t-1}, z_{t}\right) \sum_{q \in Q} T\left(q, a_{t-1}, q^{\prime}\right) b_{t-1}(q)},
\end{aligned}
$$

where the beliefs belong to the belief unit simplex

$$
\mathcal{B}=\left\{b \in[0,1]^{|Q|} \mid \sum_{q} b_{t}(q)=1, \forall t\right\} .
$$

We illustrate the notion of belief with the following example, which is more familiar to the controls community.

Example 1. Consider the following linear continuous system

$$
\left\{\begin{array}{l}
x_{t}=A_{t} x_{t-1}+B_{t} u_{t-1}+w_{t}, \\
z_{t}=C_{t} x_{t}+v_{t}
\end{array}\right.
$$

where $x \in \mathbb{R}^{n}$ are the system states, $u \in \mathbb{R}^{m}$ are the controls (actions), $z \in \mathbb{R}^{l}$ are the observations, $A_{t} \in \mathbb{R}^{n \times n}$ is the state-transition model at time $t, B_{t} \in \mathbb{R}^{n \times m}$ is the controltransition model at time $t, C_{t} \in \mathbb{R}^{l \times n}$ is the observation model at time $t$, and $w, v$ are the mutually independent noise drawn from a zero mean multivariate normal distribution, $\mathcal{N}$, with covariances $Q_{k}$ and $R_{k}$, respectively.

The Kalman filter [28] is a Bayesian estimator with states $\mu_{t \mid t}$, the a posteriori state estimate at time $t$ given observations 
up to and including at time $t$, and $\Sigma_{t \mid t}$, the a posteriori estimate covariance matrix. The Kalman filter is conceptualized as two distinct phases: "Predict" and "Update". The Kalman filter can be compactly described as

$$
\left(\mu_{t \mid t}, \Sigma_{t \mid t}\right)=f\left(\left(\mu_{t-1 \mid t-1}, \Sigma_{t-1 \mid t-1}\right), u_{t-1}, z_{t}\right),
$$

where $f$ is a nonlinear function. Indeed, for system (2), the belief is simply the states of the Kalman filter, i.e., $b_{t}=$ $\left(\mu_{t \mid t}, \Sigma_{t \mid t}\right)$, and the function $f$ is the belief update equation.

A policy in a POMDP setting is then a mapping $\pi: \mathcal{B} \rightarrow$ $A$, i.e., a mapping from the continuous belief space into the discrete and finite action space. Essentially, a policy defined over the belief space has infinite memory when mapped to the potentially infinite executions of the POMDP.

\section{PROBLEM FORMULATION}

We consider reachability, safety, and performance properties of finite POMDPs. In the following, we formally describe the latter problems.

Given an initial belief $b_{0}$ and a POMDP $\mathcal{P}$, we are concerned with estimating the set of all belief evolutions emanating from $b_{0}$, which is known as the reachable belief space.

Problem 1 (Reachable Belief Space). Given a POMDP $\mathcal{P}=$ $\left(Q, b_{0}, A, T, Z, O\right)$ as in Definition 1 with belief update (1), compute $\hat{\mathcal{R}} \subset \mathcal{B}$ such that $\mathcal{R}\left(b_{0}\right) \subseteq \hat{\mathcal{R}}$, where

$$
\mathcal{R}\left(b_{0}\right)=\left\{b \in \mathcal{B} \mid b_{t}, \text { for all } t>0, a \in A \text {, and } z \in Z\right\},
$$

is the reachable belief space.

We are also interested in studying the reachable belief space induced by a policy $\pi$.

Problem 2 (Reachable Belief Space Induced by Policy $\pi$ ). Given a POMDP $\mathcal{P}=\left(Q, b_{0}, A, T, Z, O\right)$ as in Definition 1 with belief update (1) and a policy $\pi: \mathcal{B} \rightarrow A$, compute $\hat{\mathcal{R}} \subset \mathcal{B}$ such that $\mathcal{R}^{\pi}\left(b_{0}\right) \subseteq \hat{\mathcal{R}}$, where

$$
\mathcal{R}^{\pi}\left(b_{0}\right)=\left\{b \in \mathcal{B} \mid b_{t}, \text { for all } t>0 \text { and } z \in Z\right\} .
$$

We define safety as the probability of reaching a set of unsafe states $Q_{u} \subset Q$ being less than a given constant. To this end, we use the belief states. We are interested in solving the following problem.

Problem 3 (Safety at $\tau$ ). Given a POMDP as in Definition 1, a point in time $\tau \in \mathbb{Z}_{\geq 0}$, a set of unsafe states $Q_{u} \subseteq Q$, and a safety requirement constant $\lambda$, check whether

$$
\sum_{q \in Q_{u}} b_{\tau}(q) \leq \lambda
$$

We also consider safety for all time.

Problem 4 (Safety). Given a POMDP as in Definition 1, a set of unsafe states $Q_{u} \subseteq Q$, and a safety requirement constant $\lambda$, check whether

$$
\sum_{q \in Q_{u}} b_{t}(q) \leq \lambda, \quad \forall t \in \mathbb{Z}_{\geq 0}
$$

In addition to safety, we are interested in checking whether a performance criterion is satisfied.

Problem 5 ( Performance). Given a POMDP as in Definition 1 , the reward function $R: Q \times A \rightarrow \mathbb{R}$, in which $R(q, a)$ denotes the reward of taking action a while being at state $q$, a point in time $\tau \in \mathbb{Z}_{\geq 0}$, and a performance requirement $\gamma$, check whether

$$
\sum_{s=0}^{\tau} r\left(b_{s}, a_{s}\right) \leq \gamma
$$

where $r\left(b_{s}, a_{s}\right)=\sum_{q \in Q} b_{t} R\left(q, a_{t}\right)$.

Note that, if instead of having a given $\gamma$ in Problem 5, we minimize $\gamma$ subject to a given POMDP with a reward function $R$, we can also study optimality.

\section{TREATING POMDPS AS HYBRID Systems}

Given a POMDP $\mathcal{P}$ as in Definition 1, checking whether (5) and (7) hold by solving the POMDP directly is undecidable problem [9]. In this section, we demonstrate that POMDPs can be represented as a special hybrid system [29], i.e., a discretetime switched system [30], [31], [32]. We take advantage of this representation in the sequel to formulate indirect methods for analyzing a POMDP.

According to the belief update equation (1), the current beliefs evolve according to the belief values at the previous step, actions at the previous step, and the current observations. Furthermore, the belief evolution jumps into different modes based on the actions taken. Therefore, we can represent the belief evolutions as a discrete-time switched system, where the actions $a \in A$ define the switching modes. Formally, the belief dynamics (1) can be described as

$$
b_{t}=f_{a}\left(b_{t-1}, z_{t}\right) \text {, }
$$

where $b_{t}$ denote the belief vector at time $t$ belonging to the belief unit simplex $\mathcal{B}$ and $b_{0} \in \mathcal{B}_{0} \subset \mathcal{B}$ (in particular, $\mathcal{B}_{0}$ can be a singleton) representing the set of initial beliefs (prior). In (8), $a \in A$ denote the actions that can be interpreted as the switching modes, $z \in Z$ are the observations representing inputs, and $t \in \mathbb{Z}_{\geq 1}$ denote the discrete time instances. The (rational) vector fields $\left\{f_{a}\right\}_{a \in A}$ with $f_{a}:[0,1]^{|Q|} \rightarrow[0,1]^{|Q|}$ are described as the vectors with rows

$$
f_{a}^{q^{\prime}}(b, \cdot, z)=\frac{O\left(q^{\prime}, a, z\right) \sum_{q \in Q} T\left(q, a, q^{\prime}\right) b_{t-1}(q)}{\sum_{q^{\prime} \in Q} O\left(q^{\prime}, a, z\right) \sum_{q \in Q}, T\left(q, a, q^{\prime}\right) b_{t-1}(q)},
$$

where $f_{a}^{q^{\prime}}$ denotes the $q^{\prime}$ th row of $f_{a}$.

For infinite-horizon discounted cost POMDPs, a policy then induces regions (Markov partitions) in the belief space, where an action is applied [26]. We denote the index of these mutually exclusive, connected partitions of the belief space as $\alpha \in \Gamma$, where $\Gamma$ is a finite set. Then, a policy for a POMDP can be characterized as

$$
\pi(b)= \begin{cases}a_{1}, & b \in \mathcal{B}_{\alpha_{1}} \\ \vdots & \vdots \\ a_{n}, & b \in \mathcal{B}_{\alpha_{m}},\end{cases}
$$


inducing the dynamics

$$
b_{t}= \begin{cases}f_{a_{1}}(b, z), & b \in \mathcal{B}_{\alpha_{1}} \\ \vdots & \vdots \\ f_{a_{n}}(b, z), & b \in \mathcal{B}_{\alpha_{m}},\end{cases}
$$

where $n$ and $m$ are integers. Note that the number of partitions $|\Gamma|=m$ is not necessarily less than or greater than $|A|$. Indeed, the number of $|\Gamma|=m$ depends on the method used for synthesizing the policy. For example, in the value iteration method for POMDPs, $|\Gamma|=m$ is a function of the so called $\alpha$-vectors [33].

Given the above switched system representation, we can consider two classes of problems in POMDPs:

1. Arbitrary-Policy Verification: This case corresponds to analyzing (8) under arbitrary switching with switching modes given by $a \in A$.

2. Fixed-Policy Verification: This corresponds to analyzing (8) under state-dependent switching. Indeed, the policy $\pi: \mathcal{B} \rightarrow A$ determines regions in the belief space where each mode (action) is active.

Both cases of switched systems with arbitrary switching and state-dependent switching are well-known in the systems and controls literature (see [34], [35] and references therein). Note that the second problem is well-known in the probabilistic planning [36], [37] and model checking [38] communities. For a fixed policy and a finite state space, the problem reduces to solving a linear equation system to compute the set of reachable states in the underlying Markov chain. The next example illustrates the proposed switched system representation for POMDPs with a fixed policy.

Example 2. Consider a POMDP with two states $\left\{q_{1}, q_{2}\right\}$, two actions $\left\{a_{1}, a_{2}\right\}$, and $z=\left\{z_{1}, z_{2}\right\} \in Z$. Action $a_{1}$ correspond to remaining in the same state, while action $a_{2}$ to making a transition to the other state. Observation $z_{i}$ correspond to the current state being $q_{i}$. The policy

$$
\pi= \begin{cases}a_{1}, & b \in \mathcal{B}_{1}, \\ a_{2}, & b \in \mathcal{B}_{2}\end{cases}
$$

leads to different switching modes based on whether the states belong to the regions $\mathcal{B}_{1}=\left\{b \mid 0 \leq b\left(q_{1}\right) \leq \varepsilon\right\}$ or $\mathcal{B}_{2}=\{b \mid$ $\left.\varepsilon<b\left(q_{1}\right) \leq 1\right\}$ (see Figure 1). That is, the belief update equation (8) is given by

$$
b_{t}= \begin{cases}f_{a_{1}}\left(b_{t-1}, z_{t}\right), & b \in \mathcal{B}_{1}, \\ f_{a_{2}}\left(b_{t-1}, z_{t}\right), & b \in \mathcal{B}_{2} .\end{cases}
$$

Note that the belief space is given by $\mathcal{B}=\mathcal{B}_{1} \cup \mathcal{B}_{2}=\{b \mid$ $\left.b\left(q_{1}\right)+b\left(q_{2}\right)=1\right\}$.

\section{POMDP REACHABILITY ANALYSIS USING LYAPUNOV-LIKE FUNCTIONS}

Based on the switched system representation discussed in the previous section, we can use tools from control theory to analyze the evolution of the solutions of the belief update equation including the reachable belief space without the need to solve the POMDP explicitly.

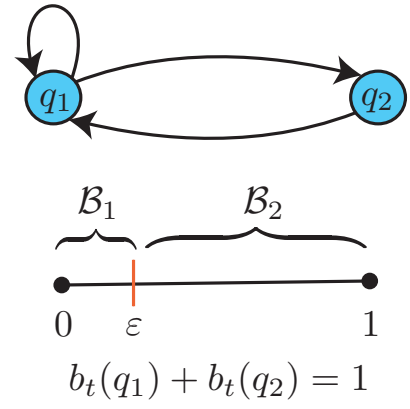

Fig. 1: An example of a POMDP with two states and the state-dependent switching modes induced by the policy (11).

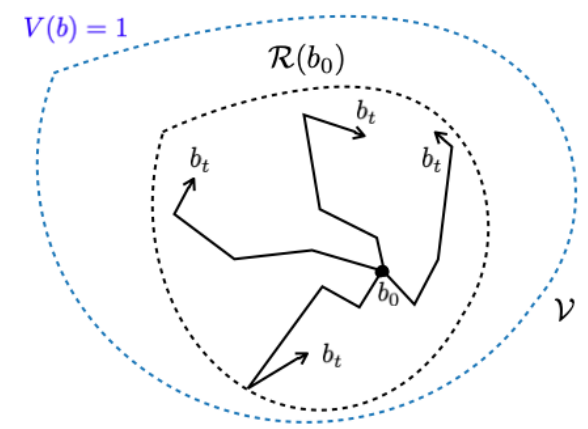

Fig. 2: Over-approximation of the reachable belief space using sub-level sets of a Lyapunov-like function.

From a control theory standpoint, estimating reachable sets, invariant sets, or regions-of-attractions (ROAs) are wellstudied problems in the literature [39]. Recent results in this area include learning ROAs using Gaussian processes and active learning [40], computation of controlled invariant sets for switching affine systems [41], ROA estimation using formal language [42], and finding invariant sets based on barrier functions [43]. In the following, we propose a basic technique for over-approximating the reachable belief spaces of POMDPs using Lyapunov-like functions.

The next theorem uses sub-level sets of Lyapunov-like functions to over-approximate the reachable belief space as illustrated in Figure 2.

Theorem 1. Given the POMDP $\mathcal{P}=\left(Q, b_{0}, A, T, Z, O\right)$ as in Definition 1, consider the belief update dynamics (8). If there exists a function $V: \mathcal{B} \rightarrow \mathbb{R}$ such that

$$
\begin{aligned}
& V\left(f_{a}\left(b_{t-1}, z\right)\right)-V\left(b_{t-1}\right)<0, \\
& \quad \text { for all } a \in A, z \in Z, b_{t-1} \in \mathcal{V},
\end{aligned}
$$

and

$$
b_{0} \in \mathcal{V}
$$

where $\mathcal{V}=\{b \in \mathcal{B} \mid V(b) \leq 1\}$, then $b_{t} \in \mathcal{V}$ for all $t \in \mathbb{Z}_{\geq 0}$, and, in particular, $\mathcal{R}\left(b_{0}\right) \subseteq \mathcal{V}$.

Proof. The proof relies on the fact that the belief trajectories point inward at the boundary of $\mathcal{V}$; hence, they remain inside $\mathcal{V}$. We prove by contradiction. Assume, at time $T \neq 0$, we 


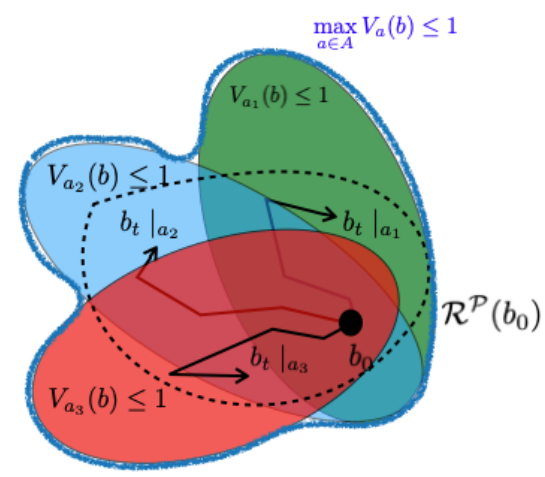

Fig. 3: Over-approximation of the reachable belief space using the sub-level sets of a set of local Lyapunov functions.

have $b_{T} \notin \mathcal{V}$ for some $a \in A$ or $z \in Z$. Thus, $V\left(b_{T}\right)>1$. Inequality (13) implies

$$
V\left(b_{t}\right)-V\left(b_{t-1}\right)<0, \text { for all } a \in A, z \in Z, b \in \mathcal{V} .
$$

Summing up the above expression from time 1 to $T$ yields

$$
\sum_{t=1}^{T}\left(V\left(b_{t}\right)-V\left(b_{t-1}\right)\right)<0, \text { for all } a \in A, z \in Z,
$$

which simplifies to

$$
V\left(b_{T}\right)-V\left(b_{0}\right)<0, \text { for all } a \in A, z \in Z, b \in \mathcal{V} .
$$

That is, $V\left(b_{T}\right)<V\left(b_{0}\right)$. In addition, from (14), we have $V\left(b_{0}\right) \leq 1$. Then, it follows that $V\left(b_{T}\right) \leq 1$, which contradict the hypothesis $b_{T} \notin \mathcal{V}$.

At this point, we prove by induction that $\mathcal{R}\left(b_{0}\right) \subseteq \mathcal{V}$. Let $\mathcal{R}_{t}\left(b_{0}\right)$ denote the reachable belief space at time $t$. At $t=$ 0 , from (13), we have $b_{0} \in \mathcal{V}$. Since $\mathcal{R}_{0}\left(b_{0}\right)=\left\{b_{0}\right\}$, then $\mathcal{R}_{0}\left(b_{0}\right) \subseteq \mathcal{V}$. At time $t=1$, from (12), we have

$$
V\left(b_{1}\right)-V\left(b_{0}\right)<0, \forall b_{0} \in \mathcal{V},
$$

which implies that $V\left(b_{1}\right)<V\left(b_{0}\right)$ for all $b_{0} \in \mathcal{V}=\{b \in$ $\left.\mathcal{B} \mid V\left(b_{0}\right) \leq 1\right\}$. Hence, $V\left(b_{1}\right)<V\left(b_{0}\right) \leq 1$ and $b_{1} \in \mathcal{V}$. In addition, $\mathcal{R}_{1}\left(b_{0}\right)=\left\{b_{0}, b_{1}\right\}$ and since $b_{0}, b_{1} \in \mathcal{V}$, then $\mathcal{R}_{1}\left(b_{0}\right) \subseteq \mathcal{V}$. Then, by induction we have for any $t=\tau$, $\tau>0, b_{\tau} \in \mathcal{V}$ and $\mathcal{R}_{\tau}\left(b_{0}\right) \subseteq \mathcal{V}$.

We remark that the function $V$ is not a Lyapunov function in the conventional sense, since we do not impose that $V(0)=0$ and $V(b)>0$ for all $b \in \mathcal{B} \backslash\{0\}$. The reason for calling $V$ a Lyapunov-like function is to make an analogy to Lyapunov based techniques for approximating reachable sets and regions of attraction, which is familiar to the controls audience [44], [45]. Henceforth, we abuse the notation and we refer to $V$ 's as Lyapunov functions.

In practice, we may have a large number of actions for a POMDP. Therefore, it may be difficult to find a single Lyapunov function $V$ that satisfies the conditions of Theorem 1. Next, we show that the computation of the Lyapunov function can be decomposed into finding a set of local Lyapunov functions for each action. This method is illustrated in Figure 3.
Theorem 2. Given the POMDP $\mathcal{P}=\left(Q, b_{0}, A, T, Z, O\right)$ as in Definition 1, consider the belief update dynamics (8). If there exist a set of functions $V_{a}: \mathcal{B} \rightarrow \mathbb{R}, a \in A$ such that

$$
\begin{aligned}
V_{a}\left(f_{a^{\prime}}\left(b_{t-1}, z\right)\right)-V_{a}\left(b_{t-1}\right)< & 0, \\
& \forall z \in Z, b_{t-1} \in \mathcal{V}_{a},
\end{aligned}
$$

for all $\left(a, a^{\prime}\right) \in A^{2}$ and

$$
b_{0} \in \mathcal{V}_{a}
$$

for all $a \in A$, where $\mathcal{V}_{a}=\left\{b \in \mathcal{B} \mid V_{a}(b) \leq 1\right\}$, then $b_{t} \in \mathcal{V}_{\text {max }}=\left\{b \in \mathcal{B} \mid \max _{a \in A} V_{a}(b) \leq 1\right\}$ for all $t \in \mathbb{Z}_{\geq 0}$, and, in particular, $\mathcal{R}\left(b_{0}\right) \subseteq \mathcal{V}_{\text {max }}$.

Proof. We show that if (15)-(16) are satisfied then the Lyapunov function $V=\max _{a \in A} V_{a}$ satisfies the conditions of Theorem 1. If (16) holds for all $a \in A$, we have $V_{a}\left(b_{0}\right) \leq 1$, which in turn implies that $\max _{a \in A} V_{a}\left(b_{0}\right) \leq 1$, as well. Thus, $V=\max _{a \in A} V_{a}$ satisfies (14). Furthermore, if (15) is satisfied for all $\left(a, a^{\prime}\right) \in A^{2}$, we have $V_{a}\left(f_{a^{\prime}}\left(b_{t-1}, z\right)\right)<V_{a}\left(b_{t-1}\right)$ for $b_{t-1} \in \mathcal{V}_{a}$, which in turn implies that $\max _{a} V_{a}\left(f_{a^{\prime}}\left(b_{t-1}, z\right)\right)<\max _{a} V_{a}\left(b_{t-1}\right)$ for $b_{t-1}$ belonging to $\max _{a} V_{a}\left(b_{t-1}\right) \leq 1$ and all $a^{\prime} \in A$. That is, (13) is satisfied with $V=\max _{a \in A} V_{a}$. Then, from Theorem 1, we have $b_{t} \in \mathcal{V}_{\text {max }}$ for all $t \in \mathbb{Z}_{\geq 0}$ and $\mathcal{R}\left(b_{0}\right) \subseteq \mathcal{V}_{\max }$.

If a policy $\pi$ is given, then we can compute a family of local Lyapunov functions. The union of the 1-sublevel sets of these functions over-approximates the reachable belief space.

Theorem 3. Given the POMDP $\mathcal{P}=\left(Q, b_{0}, A, T, Z, O\right)$ and the policy $\pi: \mathcal{B} \rightarrow A$ and an induced partitioning $\left\{\mathcal{B}_{\alpha}\right\}$ such that $\mathcal{B}=\cup_{\alpha} \mathcal{B}_{\alpha}$ as given in (9), consider the belief update dynamics (8). If there exists a family of functions $V_{\alpha}: \mathcal{B}_{\alpha} \rightarrow$ $\mathbb{R}, \alpha \in \Gamma$, such that

$$
\begin{aligned}
& V_{\alpha}\left(f_{a}\left(b_{t-1}, z\right)\right)-V_{\alpha}\left(b_{t-1}\right)<0, \\
& \text { for all } z \in Z, b_{t-1} \in \mathcal{V}_{\alpha},
\end{aligned}
$$

and

$$
b_{0} \in \mathcal{V}_{\alpha},
$$

where $\mathcal{V}_{\alpha}=\left\{b \in \mathcal{B}_{\alpha} \mid V_{\alpha}(b) \leq 1\right\}$, then $b_{t} \in \cup_{\alpha} \mathcal{V}_{\alpha}$ for all $t \in \mathbb{Z}_{\geq 0}$, and, in particular, $\mathcal{R}\left(b_{0}\right) \subseteq \cup_{\alpha \in \Gamma} \mathcal{V}_{\alpha}$.

Proof. From Theorem 1, we can infer that, for each fixed $a \in A$, we have $\mathcal{R}_{a}\left(b_{0}\right) \subseteq \mathcal{V}_{\alpha}$, where $\mathcal{R}_{a}\left(b_{0}\right)$ is the reachable belief space under action $a$ starting at $b_{0}$. Since the support of each $V_{\alpha_{i}}$ is $\mathcal{B}_{\alpha_{i}}, \mathcal{V}_{\alpha_{i}} \subseteq \mathcal{B}_{\alpha_{i}}, \cup_{\alpha \in \Sigma} \mathcal{B}_{\alpha}=\mathcal{B}$, then $\cup_{\alpha \in \Sigma} \mathcal{V}_{\alpha}=\mathcal{V} \subseteq \mathcal{B}$. Furthermore, since $\mathcal{R}_{a}\left(b_{0}\right) \subseteq \mathcal{V}_{\alpha}$, then $\cup_{a \in A} \mathcal{R}_{a}\left(b_{0}\right)=\mathcal{R}\left(b_{0}\right) \subseteq \cup_{\alpha \in \Gamma} \mathcal{V}_{\alpha}$

We remark at the end of this section that the existence of a policy $\pi$ for a POMDP removes all non-determinism and partial observability, resulting in an induced Markov chain. In this case, in [46], a method based on Foster-Lyapunov functions was used for analyzing Markov chains, which may be also adapted to verify POMDPs with a fixed policy. 


\section{POMDP SAFETY VERIFICATION}

\section{USING BARRIER CERTIFICATES}

In the following, we show how we can use barrier certificates to verify properties of the switched systems induced by a POMDP. We begin by verifying safety for a given POMDP.

Let us define the following unsafe set

$$
\mathcal{B}_{u}^{s}=\left\{b \in \mathcal{B} \mid \sum_{q \in Q_{u}} b_{\tau}(q)>\lambda\right\},
$$

which is the complement of (5).

Theorem 4. Given the POMDP $\mathcal{P}=\left(Q, b_{0}, A, T, Z, O\right)$ as in Definition 1, consider the belief update dynamics (8). Given a set of initial beliefs $\mathcal{B}_{0} \subset[0,1]^{|Q|}$, an unsafe set $\mathcal{B}_{u}^{s}$ as given in (19) $\left(\mathcal{B}_{0} \cap \mathcal{B}_{u}^{s}=\emptyset\right)$, and a constant $\tau \in \mathbb{Z}_{\geq 0}$, if there exists a function $B: \mathbb{Z} \times \mathcal{B} \rightarrow \mathbb{R}$ called the barrier certificate such that

$$
\begin{aligned}
& B\left(\tau, b_{\tau}\right)>0, \quad \forall b_{\tau} \in \mathcal{B}_{u}^{s}, \\
& B\left(0, b_{0}\right)<0, \quad \forall b_{0} \in \mathcal{B}_{0},
\end{aligned}
$$

and

$$
\begin{aligned}
& B\left(t, f_{a}\left(b_{t-1}, z\right)\right)-B\left(t-1, b_{t-1}\right) \leq 0, \\
& \forall t \in\{1,2, \ldots, \tau\}, \forall a \in A, \forall z \in Z, \forall b \in \mathcal{B},
\end{aligned}
$$

then there exist no solution of the belief update equation (8) such that $b_{0} \in \mathcal{B}_{0}$, and $b_{\tau} \in \mathcal{B}_{u}^{s}$ for all $a \in A$.

Proof. Please refer to the proof of Theorem 1 in [47] noting that $\Theta=\emptyset$.

The above theorem provides conditions under which the POMDP is guaranteed to be safe. The next result brings forward a set of conditions, which verifies whether the performance criterion (7) is satisfied. This is simply carried out by making the unsafe set time-dependent (a tube).

Corollary 1. Given the POMDP $\mathcal{P}=\left(Q, b_{0}, A, T, Z, O\right)$ as in Definition 1, consider the belief update dynamics (8) and the performance criterion $\gamma$ as given by (7). Let $\tilde{\gamma}: \mathbb{Z}_{\geq 0} \rightarrow \mathbb{R}$ satisfying

$$
\sum_{s=0}^{\tau} \tilde{\gamma}(s) \leq \gamma
$$

Given a set of initial beliefs $\mathcal{B}_{0} \subset \mathcal{B}$, an unsafe set

$$
\mathcal{B}_{u}^{o}=\left\{(t, b) \mid r\left(b_{t}, a_{t}\right)>\gamma(t)\right\},
$$

and a constant $\tau \in \mathbb{Z}_{\geq 0}$, if there exists a function $B: \mathbb{Z} \times \mathcal{B} \rightarrow$ $\mathbb{R}$ such that (20)-(22) are satisfied with $\mathcal{B}_{u}^{o}$ instead of $\mathcal{B}_{u}^{s}$, then for all $b_{0} \in \mathcal{B}_{0}$ the performance criterion (7) holds.

Proof. Please refer to the proof of Corollary 1 in [47] noting that $\Theta=\emptyset$.

The technique used in Corollary 1 is analogous to the one used in [48], [49] for bounding (time-averaged) functional outputs of systems described by partial differential equations. The method proposed here, however, can be used for a large class of discrete time systems and the belief update equation is a special case that is of our interest.
In practice, we may have a large number of actions. Then, finding a barrier certificate that satisfies the conditions of Theorem 4 becomes computationally prohibitive. In the next result, we show how the calculation of the barrier certificate can be decomposed into finding a set of barrier certificates for each action and then taking the convex hull of them.

Theorem 5. Given the POMDP $\mathcal{P}=\left(Q, b_{0}, A, T, Z, O\right)$ as in Definition 1, consider the belief update dynamics (8). Given a safety constraint as described in (5) with a safety requirement $\lambda$, and a point in time $\tau \in \mathbb{Z}_{\geq 0}$, if there exists a set of functions $B_{a}: \mathbb{Z} \times \mathcal{B} \rightarrow \mathbb{R}, a \in A$, such that

$$
B_{a}\left(\tau, b_{\tau}\right)>0, \quad \forall b_{\tau} \in \mathcal{B}_{u}^{s}, \quad \forall a \in A,
$$

with $\mathcal{B}_{u}^{s}$ as described in (19),

$$
B_{a}\left(0, b_{0}\right)<0, \quad \text { for } \quad b_{0}=p_{0}, \quad \forall a \in A,
$$

and

$$
\begin{aligned}
& B_{a}\left(t, f_{a^{\prime}}\left(b_{t-1}, z\right)\right)-B_{a}\left(t-1, b_{t-1}\right) \leq 0, \\
& \forall t \in\{1,2, \ldots, \tau\}, \forall\left(a, a^{\prime}\right) \in A^{2}, \forall z \in Z, \forall b \in \mathcal{B},
\end{aligned}
$$

then the safety requirement $\lambda$ is satisfied, i.e., inequality (5) holds. Furthermore, the overall barrier certificate is given by $B=\operatorname{co}\left\{B_{a}\right\}_{a \in A}$.

Proof. We show that if (25)-(27) are satisfied then the barrier certificate $B=\operatorname{co}\left\{B_{a}\right\}_{a \in A}$ satisfies the conditions of Theorem 4. For each $a \in A$, we multiply both sides of (25) with a constant $\alpha_{a}$ such that $\sum_{a \in A} \alpha_{a}=1$. We obtain

$$
\sum_{a \in A} \alpha_{a} B_{a}\left(\tau, b_{\tau}\right)>0, \quad \forall b_{\tau} \in \mathcal{B}_{u}^{s}
$$

which implies that

$$
B\left(\tau, b_{\tau}\right)=\operatorname{co}\left\{B_{a}\left(\tau, b_{\tau}\right)\right\}_{a \in A}>0, \forall b_{\tau} \in \mathcal{B}_{u}^{s} .
$$

Therefore, (25) is satisfied with $B=\operatorname{co}\left\{B_{a}\right\}_{a \in A}$. Similarly, we can show that if (26) is satisfied, $B=\operatorname{co}\left\{B_{a}\right\}_{a \in A}$ satisfies (21). Multiplying both sides of (27) with a constant $\alpha_{a}$ such that $\sum_{a \in A} \alpha_{a}=1$ and summing over them yields

$$
\begin{aligned}
& \sum_{a \in A} \alpha_{a}\left(B_{a}\left(t, f_{a^{\prime}}\left(b_{t-1}, z\right)\right)-B_{a}\left(t-1, b_{t-1}\right)\right) \\
& =\sum_{a \in A} \alpha_{a} B_{a}\left(t, f_{a^{\prime}}\left(b_{t-1}, z\right)\right)-\sum_{a \in A} \alpha_{a} B_{a}\left(t-1, b_{t-1}\right) \leq 0, \\
& \forall t \in\{1,2, \ldots, \tau\}, \forall z \in Z, \forall a^{\prime} \in A, \forall b \in \mathcal{B} .
\end{aligned}
$$

which implies that (22) is satisfied for $B=\operatorname{co}\left\{B_{a}\right\}_{a \in A}$. Therefore, from Theorem 4 , we conclude that the safety requirement $\lambda$ is satisfied.

The efficacy of the above result is that we can search for each action-based barrier certificate $B_{a}, a \in A$, independently or in parallel and then verify whether the overall POMDP satisfies a pre-specified safety requirement (see Fig. 4 for an illustration).

Next, we demonstrate that, if a policy is given, the search for the barrier certificate can be decomposed into the search for a set of local barrier certificates. We denote by $a_{i}$ the action active in the partition $\mathcal{B}_{i}$. 
Theorem 6. Given the POMDP $\mathcal{P}=\left(Q, b_{0}, A, T, Z, O\right)$ as in Definition 1, consider the belief update dynamics (8). Given a safety constraint as described in (5) with a safety requirement $\lambda$, a point in time $\tau \in \mathbb{Z}_{\geq 0}$, and a policy $\pi: \mathcal{B} \rightarrow A$ as described in (9), if there exists a set of functions $B_{i}: \mathbb{Z} \times \mathcal{B}_{i} \rightarrow$ $\mathbb{R}, i \in\{1,2, \ldots, N\}$, such that

$$
B_{i}\left(\tau, b_{\tau}\right)>0, \quad \forall b_{\tau} \in \mathcal{B}_{u}^{o} \cap \mathcal{B}_{i}, \quad i \in\{1,2, \ldots, m\},
$$

with $\mathcal{B}_{u}^{o}$ as described in (19),

$$
B_{i}\left(0, b_{0}\right)<0, \quad \text { for } \quad b_{0}=p_{0}, \quad i \in\{1,2, \ldots, m\},
$$

and

$$
\begin{aligned}
B_{i}\left(t, f_{a_{i}}\left(b_{t-1}, z\right)\right)-B_{i}\left(t-1, b_{t-1}\right) & \leq 0 \\
\forall t \in\{1,2, \ldots, \tau\}, \quad \forall z & \in Z, \quad \forall b \in \mathcal{B}_{i}, \\
& i \in\{1,2, \ldots, m\},
\end{aligned}
$$

then the safety requirement $\lambda$ is satisfied, i.e., inequality (5) holds. Furthermore, the overall barrier certificate is given by $B=\operatorname{co}\left\{B_{i}\right\}_{i=1}^{m}$.

Proof. We demonstrate that if (28)-(30) are satisfied then the barrier certificate $B=\operatorname{co}\left\{B_{i}\right\}_{i=1}^{m}$ satisfies the conditions of Theorem 4. For each $i \in\{1,2, \ldots, m\}$, we multiply both sides of (28) with a constant $\alpha_{i}$ such that $\sum_{i=1}^{m} \alpha_{i}=1$. We obtain

$$
\sum_{i=1}^{m} \alpha_{i} B_{i}\left(\tau, b_{\tau}\right)>0, \quad \forall b_{\tau} \in \cup_{i=1}^{m}\left(\mathcal{B}_{u}^{s} \cap \mathcal{B}_{i}\right) .
$$

Since the support of each $B_{i}$ is $\mathcal{B}_{i}, \cup_{i=1}^{m} \mathcal{B}_{i}=\mathcal{B}$, and $\mathcal{B}_{u}^{s} \subset \mathcal{B}$, we have $\cup_{i=1}^{m}\left(\mathcal{B}_{u}^{s} \cap \mathcal{B}_{i}\right)=\mathcal{B}_{u}^{s} \cap \mathcal{B}=\mathcal{B}_{u}^{s}$. Hence,

$$
B\left(\tau, b_{\tau}\right)=\operatorname{co}\left\{B_{i}\left(\tau, b_{\tau}\right)\right\}_{i=1}^{N}>0, \forall b_{\tau} \in \mathcal{B}_{u}^{s} .
$$

Therefore, (20) is satisfied with $B=\operatorname{co}\left\{B_{i}\right\}_{i=1}^{m}$. Similarly, we can show that if (29) is satisfied, $B=\operatorname{co}\left\{B_{i}\right\}_{i=1}^{m}$ satisfies (21). Multiplying both sides of (30) with constants $\alpha_{i}$ such that $\sum_{i=1}^{m} \alpha_{i}=1$ and summing over them gives

$$
\begin{aligned}
& \sum_{i=1}^{m} \alpha_{i}\left(B_{i}\left(t, f_{a_{i}}\left(b_{t-1}, z\right)\right)-B_{i}\left(t-1, b_{t-1}\right)\right) \\
& =\sum_{i=1}^{m} \alpha_{i} B_{i}\left(t, f_{a_{i}}\left(b_{t-1}, z\right)\right)-\sum_{i=1}^{m} \alpha_{i} B_{i}\left(t-1, b_{t-1}\right) \leq 0, \\
& \forall t \in\{1,2, \ldots, \tau\}, \forall z \in Z, \forall b \in \mathcal{B} .
\end{aligned}
$$

which implies that (22) is satisfied for $B=\operatorname{co}\left\{B_{i}\right\}_{i=1}^{m}$. Therefore, from Theorem 4 , we conclude that the safety requirement $\lambda$ is satisfied.

We proposed two techniques for decomposing the construction of the barrier certificates and checking given safety requirements. Our method relied on barrier certificates that take the form of the convex hull of a set of local barrier certificates (see similar results in [50], [51]). Though the convex hull barrier certificate may introduce a level of conservatism, it is computationally easier to find (as will be discussed in more detail in the next section).

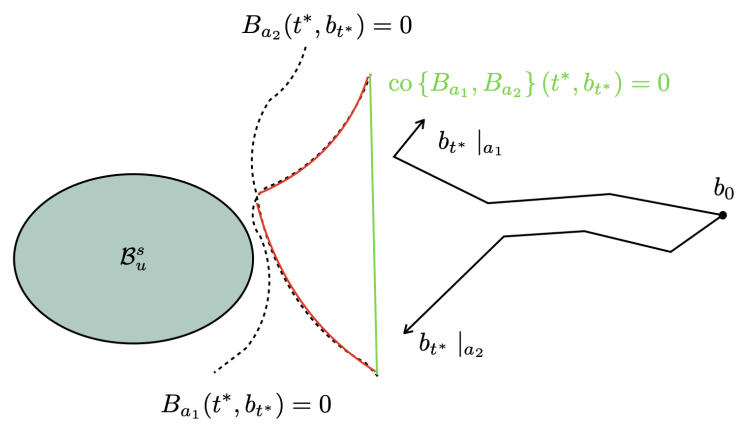

Fig. 4: Decomposing the barrier certificate computation for a POMDP with two actions $a_{1}$ and $a_{2}$ : the zero-level sets of $B_{a_{1}}$ and $B_{a_{2}}$ at trial $\tau$ separate the evolutions of the hypothesis beliefs starting at $b_{0}$ from $\mathcal{B}_{u}^{o}$. The green line illustrate the zero-level set of the barrier certificate formed by taking the convex hull of $B_{a_{1}}$ and $B_{a_{2}}$.

\section{ViI. Computational Method based on SUM-OF-SQUARES PROGRAMMING}

In this section, we present a set of SOS programs that can be used to find the Lyapunov functions used for reachability analysis and the barrier certificates used for safety and performance verification. For a brief introduction to SOS programming and some related results, see Appendix A.

In order to formulate SOS programs, we need the problem data to be polynomial or rational. Fortunately, the belief update equation (1) is a rational function in the belief states $b_{t}(q), q \in$ $Q$

$$
\begin{aligned}
& b_{t}\left(q^{\prime}\right)=\frac{M_{a}\left(b_{t-1}\left(q^{\prime}\right), z\right)}{N_{a}\left(b_{t-1}\left(q^{\prime}\right), z\right)} \\
= & \frac{O\left(q^{\prime}, a_{t-1}, z_{t}\right) \sum_{q \in Q} T\left(q, a_{t-1}, q^{\prime}\right) b_{t-1}(q)}{\sum_{q^{\prime} \in Q} O\left(q^{\prime}, a_{t-1}, z_{t}\right) \sum_{q \in Q} T\left(q, a_{t-1}, q^{\prime}\right) b_{t-1}(q)},
\end{aligned}
$$

where $M_{a}$ and $N_{a}$ are linear and positive functions of $b$.

Moreover, the safe, and the performance objectives described by (5) and (7), respectively, and the belief simplex are all semi-algebraic sets, since they can be described by polynomial inequalities/equalities.

Throughout this section, we also assume that the Lyapunov functions and the barrier certificates are parametrized as polynomial functions of $b$. Hence, the level sets of those functions are semi-algebraic sets, as well.

At this stage, we are ready to present conditions based on SOS programs to find a Lyapunov function for POMDPs and thus over-approximate the reachable belief space.

Corollary 2. Given the POMDP $\mathcal{P}=\left(Q, b_{0}, A, T, Z, O\right)$ as in Definition 1, consider the belief update dynamics (31). If there exist a set of functions $V \in P[b]$ of degree $d$ and $p \in \Sigma[b]$, and $a$ constant $c>0$ such that

$$
\begin{aligned}
&-N_{a}^{d}\left(b_{t-1}, z\right)\left(V\left(\frac{M_{a}\left(b_{t-1}, z\right)}{N_{a}\left(b_{t-1}, z\right)}\right)-V\left(b_{t-1}\right)\right) \\
&-p\left(b_{t-1}\right)\left(1-V\left(b_{t-1}\right)\right)-c \in \Sigma\left[b_{t-1}\right],
\end{aligned}
$$

for all $a \in A$ and $z \in Z$, and

$$
1-V\left(b_{0}\right) \geq 0,
$$


then $b_{t} \in \mathcal{V}=\{b \in \mathcal{B} \mid V(b) \leq 1\}$ for all $t \in \mathbb{Z}_{\geq 0}$, and, in particular, $\mathcal{R}\left(b_{0}\right) \subseteq \mathcal{V}$.

Proof. We show that if (32) and (33) hold, then (13) and (14) are, respectively, satisfied. Condition (33) implies that (14) holds. Furthermore, condition (13) for system (31) can be rewritten as

$$
-\left(V\left(\frac{M_{a}\left(b_{t-1}, z\right)}{N_{a}\left(b_{t-1}, z\right)}\right)-V\left(b_{t-1}\right)\right)>0, \forall a \in A, \forall z \in Z .
$$

Since $\mathcal{V}=\{b \mid 1-V(b) \geq 0\}$ is a semi-algebraic set, we use Propositions 1 and 2 in Appendix A to obtain

$$
\begin{aligned}
& -\left(V\left(\frac{M_{a}\left(b_{t-1}, z\right)}{N_{a}\left(b_{t-1}, z\right)}\right)-V\left(b_{t-1}\right)\right) \\
& -p\left(b_{t-1}\right)\left(1-V\left(b_{t-1}\right)\right)-c \in \Sigma\left[b_{t-1}\right], \forall a \in A, \forall z \in Z .
\end{aligned}
$$

for $p \in \Sigma[b]$ and $c>0$. Given that $N_{a}\left(b_{t-1}, z\right)$ is a positive polynomial of degree one, we can relax the above inequality into a sum-of-squares condition given by

$$
\begin{aligned}
& -N_{a}^{d}\left(b_{t-1}, z\right)\left(V\left(\frac{M_{a}\left(b_{t-1}, z\right)}{N_{a}\left(b_{t-1}, z\right)}\right)-V\left(b_{t-1}\right)\right) \\
& -p\left(b_{t-1}\right)\left(1-V\left(b_{t-1}\right)\right)-c \in \Sigma\left[b_{t-1}\right], \forall a \in A, \forall z \in Z .
\end{aligned}
$$

Hence, if (32) holds, then (13) is satisfied, as well. From Theorem 1, we infer that $b_{t} \in \mathcal{V}=\{b \in \mathcal{B} \mid V(b) \leq 1\}$ for all $t \in \mathbb{Z}_{\geq 0}$, and $\mathcal{R}\left(b_{0}\right) \subseteq \mathcal{V}$.

The set $\mathcal{V}$ provides an over-approximation of the reachable belief space $\mathcal{R}\left(b_{0}\right)$. Indeed, we can tighten the overapproximation by solving the following optimization problem:

$$
\begin{gathered}
\min _{V \in P[b], \gamma>0, p \in \Sigma[b], c>0} \gamma \\
V\left(b_{0}\right)-\gamma \leq 0 \\
-V\left(f_{a}(b, z)\right)+V(b)-p(b)(\gamma-V(b))-c \in \Sigma[b],
\end{gathered}
$$

for all $a \in A$ and $z \in Z$. The above optimization is bilinear in the variables $V, \gamma$, and $p$. However, If $p$ is fixed, the problem becomes convex. Similarly, when $V$ and $\gamma$ are fixed, the optimization problem is convex in $p$. Optimization problems similar to (34) have been proposed in the literature for estimating the region-of-attraction of polynomial (continuous) systems [44].

We can computationally implement Theorem 2 by solving $|A|$ optimization problems

$$
\begin{gathered}
\min _{V_{a} \in P[b], \gamma>0, p_{a} \in \Sigma[b], c_{a}>0} \gamma_{a} \\
V_{a}\left(b_{0}\right)-\gamma \leq 0, \\
-V_{a}\left(f_{a^{\prime}}(b, z)\right)+V_{a}(b)-p_{a}(b)\left(\gamma_{a}-V_{a}(b)\right) \in \Sigma[b],
\end{gathered}
$$

for all $z \in Z$ and $a^{\prime} \in A$ and then computing the level set via $\max _{a \in A} V_{a}(b) \leq \gamma$.

Furthermore, we can implement Theorem 3 by solving $|\Gamma|$ optimization problems

$$
\begin{gathered}
\min _{V_{\alpha} \in P[b], \gamma_{\alpha}>0, R_{\alpha} \in \Sigma[b]} \gamma_{\alpha} \\
V_{\alpha}\left(b_{0}\right)-\gamma_{\alpha} \leq 0, \\
-V_{\alpha}\left(f_{a}(b, z)\right)+V_{\alpha}(b)-R_{\alpha}(b)\left(\gamma_{\alpha}-V_{\alpha}(b)\right) \in \Sigma[b],
\end{gathered}
$$

for all $z \in Z$ in parallel and then computing $\cup_{\alpha \in \Gamma}\left\{b \mid V_{\alpha}(b) \leq\right.$ $\left.\gamma_{\alpha}\right\}$.

Next, we present sum-of-squares programs for finding the barrier certificates and check safety and performance. We begin by an SOS formulation for Theorem 4 .

Corollary 3. Given the POMDP $\mathcal{P}=\left(Q, b_{0}, A, T, Z, O\right)$ as in Definition 1, consider the belief update dynamics (31). Given a safety constraint as described in (5) with a safety requirement $\lambda$, and a point in time $\tau \in \mathbb{Z}_{>0}$, if there exist polynomial functions $B \in P[t, b]$ of degree $d$ and $p^{f} \in \Sigma[b]$, and constants $s_{1}, s_{2}>0$ such that

$$
\begin{aligned}
B\left(\tau, b_{\tau}\right)+p^{f}\left(b_{\tau}\right) & \left(\sum_{q \in Q_{u}} b_{\tau}(q)-\lambda\right)-s_{1} \in \Sigma\left[b_{\tau}\right], \\
& -B\left(0, b_{0}\right)-s_{2}>0,
\end{aligned}
$$

and

$$
\begin{gathered}
-N_{a}\left(b_{t-1}\right)^{d}\left(B\left(t, \frac{M_{a}\left(b_{t-1}, z\right)}{N_{a}\left(b_{t-1}, z\right)}\right)-B\left(t-1, b_{t-1}\right)\right) \\
\in \Sigma\left[t, b_{t-1}\right], \forall t \in\{1,2, \ldots, \tau\}, z \in Z, a \in A,
\end{gathered}
$$

then there exists no solution of (8) such that $b_{\tau} \in \mathcal{B}_{u}^{s}$ and, hence, the safety requirement is satisfied.

Proof. Please refer to the proof of Corollary 2 in [47] noting that $\Theta=\emptyset$.

Similarly, we can formulate SOS feasibility conditions for checking the inequalities in Theorem 5 .

Corollary 4. Given the $P O M D P \mathcal{P}=\left(Q, b_{0}, A, T, Z, O\right)$ as in Definition 1, consider the belief update dynamics (31). Given a safety constraint as described in (5) with a safety requirement $\lambda$, and a point in time $\tau \in \mathbb{Z}_{\geq 0}$, if there exist polynomial functions $B_{a} \in P[t, b], a \in A$, of degree $d$ and $p_{a}^{f} \in \Sigma[b]$, $a \in A$, and constants $s_{a}^{1}, s_{a}^{2}>0, a \in A$, such that

$$
\begin{gathered}
B_{a}\left(\tau, b_{\tau}\right)+p_{a}^{f}\left(b_{\tau}\right)\left(\sum_{q \in Q_{u}} b_{\tau}(q)-\lambda\right) \\
-s_{a}^{1} \in \Sigma\left[b_{\tau}\right], \quad a \in A, \\
-B_{a}\left(0, b_{0}\right)-s_{a}^{2}>0, \quad a \in A,
\end{gathered}
$$

and

$$
\begin{aligned}
& -N_{a}\left(b_{t-1}\right)^{d}\left(B_{a}\left(t, \frac{M_{a^{\prime}}\left(b_{t-1}, z\right)}{N_{a^{\prime}}\left(b_{t-1}, z\right)}\right)-B_{a}\left(t-1, b_{t-1}\right)\right) \\
\in & \Sigma\left[t, b_{t-1}\right], \forall t \in\{1,2, \ldots, \tau\}, z \in Z, \quad\left(a, a^{\prime}\right) \in A^{2}, \quad(42)
\end{aligned}
$$

then there exists no solution of (31) such that $b_{\tau} \in \mathcal{B}_{u}^{s}$ and, hence, the safety requirement is satisfied.

We assume that a policy in the form of (9) assigns actions to semi-algebraic partitions of the hypothesis belief space $\mathcal{B}$ described as

$$
\mathcal{B}_{i}=\left\{b \in \mathcal{B} \mid g_{i}(b) \leq 0\right\}, \quad i \in\{1,2, \ldots, m\} .
$$

We then have the following SOS formulation for Theorem 6 using Positivstellensatz (Proposition 1 in Appendix A). 
Corollary 5. Given the POMDP $\mathcal{P}=\left(Q, b_{0}, A, T, Z, O\right)$ as in Definition 1, consider the belief update dynamics (31). Given a safety constraint as described in (5) with a safety requirement $\lambda$, and a point in time $\tau \in \mathbb{Z}_{\geq 0}$, a policy $\pi: \mathcal{B} \rightarrow A$ as described in (9), if there exist polynomial functions $B_{i} \in P[t, b]$, $i \in\{1,2, \ldots, m\}$, of degree $d, p_{i}^{l_{1}} \in \Sigma[b], i \in\{1,2, \ldots, m\}$, $p_{i}^{l_{2}} \in \Sigma[b], i \in\{1,2, \ldots, m\}, p_{i}^{l_{3}} \in \Sigma[b], i \in\{1,2, \ldots, m\}$, and $p_{i}^{f} \in \Sigma[b], i \in\{1,2, \ldots, m\}$, and constants $s_{i}^{1}, s_{i}^{2}>0$, $i \in\{1,2, \ldots, m\}$, such that

$$
\begin{gathered}
B_{i}\left(\tau, b_{\tau}\right)+p_{i}^{f}\left(b_{\tau}\right)\left(\sum_{q \in Q_{u}} b_{\tau}(q)-\lambda\right)+p_{i}^{l_{1}}\left(b_{\tau}\right) g_{i}\left(b_{\tau}\right) \\
-s_{i}^{1} \in \Sigma\left[b_{\tau}\right], \quad i \in\{1,2, \ldots, m\}, \quad(44) \\
-B_{i}\left(0, b_{0}\right)+p_{i}^{l_{2}}\left(p_{0}\right) g_{i}\left(p_{0}\right)-s_{i}^{2}>0, \quad i \in\{1,2, \ldots, m\},
\end{gathered}
$$

and

$$
\begin{gathered}
-N_{a}\left(b_{t-1}\right)^{d}\left(B_{i}\left(t, \frac{M_{a}\left(b_{t-1}, z\right)}{N_{a}\left(b_{t-1}, z\right)}\right)-B_{i}\left(t-1, b_{t-1}\right)\right) \\
+p_{i}^{l_{3}}\left(b_{t-1}\right) g_{i}\left(b_{t-1}\right) \in \Sigma\left[t, b_{t-1}\right], \forall t \in\{1,2, \ldots, \tau\}, \\
z \in Z, a \in A, \quad i \in\{1,2, \ldots, m\}, \quad \text { (46) }
\end{gathered}
$$

then there exists no solution of (31) such that $b_{\tau} \in \mathcal{B}_{u}^{s}$ and, hence, the safety requirement is satisfied.

Checking whether the performance holds can also be cast into sum-of-squares programs. To this end, we assume the reward function is a polynomial (or can be approximated by a polynomial $^{1}$ ) in beliefs, i.e., $R \in P[b]$.

The following Corollary can be derived using similar arguments as the proof of Corollary 3 .

Corollary 6. Given the POMDP $\mathcal{P}=\left(Q, b_{0}, A, T, Z, O\right)$ as in Definition 1, consider the belief update dynamics (31). Given a constant $\tau \in \mathbb{Z}_{\geq 0}$ and the performance requirement (7), if there exist polynomial functions $\tilde{\gamma} \in P[t]$ characterizing the unsafe set (24), $B \in P[t, b]$ with degree $d, p_{q}^{u} \in \Sigma[b], q \in Q_{u}$, $p_{i}^{0} \in \Sigma[b], i=1,2, \ldots, n_{0}$, and constants $s_{1}, s_{2}>0$ such that (23), and (37)-(39) are satisfied, then the performance criterion (7) holds.

\section{CASE STUdies}

In this section, we illustrate the proposed method using two examples. In the first example, we study a toy example of ad scheduling scenario in social media using a POMDP model. We illustrate how the over-approximation method based on local Lyapunov functions introduced in Section $\mathrm{V}$ can be used to predict the outcome of an ad scheduling policy. In the second example, we compare the teaching performance of two recently proposed machine teaching algorithms (myopic and Ada-L) [53] and show the safety verification method presented in Section VI can be used to show that myopic teaching has poor performance while Ada-L has guaranteed convergence.

\footnotetext{
${ }^{1}$ This assumption is realistic, since the beliefs belong to a bounded set (a unit simplex) and by Stone-Weierstrass theorem any continuous function defined on a bounded domain can be uniformly approximated arbitrary close by a polynomial [52].
}

The numerical experiments are carried out on a MacBook Pro 2.9 GHz Intel Core i5 and 8GB of RAM.

\section{A. Interactive Advertisement Scheduling}

In personalized live social media, a website's revenue depends on user engagement, which is closely related to the user's interest to the streamed online contents [54]. User interest evolves probabilistically with the online contents and the ads inserted [55]. Furthermore, user interest to online content is not directly observable but can only be inferred from the number of views or the number of likes during a given time interval [56]. The broadcaster's objective is to estimate user interest and schedule relevant ads from time to time to maintain high user interest and engagement.

Such interactive ads scheduling can be represented as a sequential decision-making problem under partial observability [54], where a POMDP $\mathcal{P}=\left(Q, p_{0}, A, T, Z, O\right)$ can describe how the user's interest evolves. Each state $q \in Q$ represents different levels of interest where $p_{0}$ denotes the initial interest distribution. The actions available to the broadcaster are to insert different ads or continue with the live stream. The transition $T\left(q, a, q^{\prime}\right)$ describes how a user's interest may evolve depending on the ads inserted or the content of live stream when no ads is scheduled. The observation $Z$ denotes the number of likes in a given time interval, which can be abstracted into a finite number of categories. The observation function $O(q, z)$ denotes the probability of observing $z$ likes when the user's interest level is at $q$. The transition and observation functions can be obtained from data [54].

We consider a concrete POMDP model $\mathcal{P}$ where the topology of the underlying MDP is as shown in Figure 5. The user interest has three levels - low, medium, and high. The initial probability distribution of the user interest is $p_{0}\left(q_{1}\right)=1$, i.e., the user initially has low interest. The actions available are $A=\left\{a_{0}, a_{1}\right\}$, where $a_{0}$ denotes no ads and $a_{1}$ denotes scheduling ads. The transition probabilities $T$ with respect to each action are as shown in (47).

$$
T_{a_{0}}=\left[\begin{array}{lll}
0.8, & 0.2, & 0.1 \\
0.1, & 0.7, & 0.2 \\
0.1, & 0.1, & 0.7
\end{array}\right], T_{a_{1}}=\left[\begin{array}{lll}
0.5, & 0.3, & 0.2 \\
0.3, & 0.6, & 0.2 \\
0.2, & 0.1, & 0.6
\end{array}\right] .
$$

The observations are number of likes in a give time interval and can be represented by a Poisson process [54]. To have a finite number of observations, we have $Z=\left\{z_{1}, z_{2}, z_{3}\right\}$ to denote low, medium, and high number of likes with the thresholds $\gamma_{1}$ and $\gamma_{2}$. The observation functions are then defined as follows.

$$
\begin{aligned}
& O\left(q_{i}, z_{1}\right)=\sum_{j \leq \gamma_{1}} \frac{\lambda_{i}^{j} \exp \left(-\lambda_{i}\right)}{j !}, \\
& O\left(q_{i}, z_{2}\right)=\sum_{\gamma_{1}<j \leq \gamma_{2}} \frac{\lambda_{i}^{j} \exp \left(-\lambda_{i}\right)}{j !}, \\
& O\left(q_{i}, z_{3}\right)=\sum_{j>\gamma_{2}} \frac{\lambda_{i}^{j} \exp \left(-\lambda_{i}\right)}{j !},
\end{aligned}
$$

where $\lambda_{i}$ represents the rate of Poisson process when the interest is in state $q_{i}$. In this particular example, $\lambda_{1}=2$, 


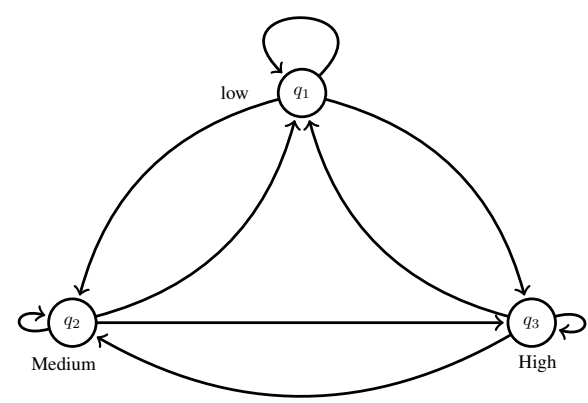

Fig. 5: The underlying MDP in Example I

$\lambda_{2}=4, \lambda_{3}=6, \gamma_{1}=3$ and $\gamma_{2}=6$. Then from (48), it can be found that

$O\left(q_{1}, z_{1}\right)=0.8571, O\left(q_{1}, z_{2}\right)=0.1383, O\left(q_{1}, z_{3}\right)=0.0046$,
$O\left(q_{2}, z_{1}\right)=0.4335, O\left(q_{2}, z_{2}\right)=0.4559, O\left(q_{2}, z_{3}\right)=0.1106$,
$O\left(q_{3}, z_{1}\right)=0.1512, O\left(q_{3}, z_{2}\right)=0.4551, O\left(q_{3}, z_{3}\right)=0.3937$.

In this example, we study the reachable belief space given the following policy

$$
\pi(b)= \begin{cases}a_{0}, & b \in \mathcal{B}_{1}, \\ a_{1}, & b \in \mathcal{B}_{2},\end{cases}
$$

where $\mathcal{B}_{1}=\left\{b \mid b\left(q_{1}\right)+b\left(q_{2}\right) \leq 0.5\right\}$ and $\mathcal{B}_{2}=\left\{b \mid b\left(q_{1}\right)+\right.$ $\left.b\left(q_{2}\right)>0.5\right\}$. That is, if the probability of the user's interest being low or medium is less than 0.5 show ads, and if the probability of the user's interest being high is greater than 0.5 show no ads (see Figure 6).

In this example, we assume we are not sure about the interest level of the user. Therefore, we assign equal initial probability to all three states $\left(b_{0}\left(q_{1}\right)=b_{0}\left(q_{2}\right)=b_{0}\left(q_{3}\right)=\right.$ $1 / 3)$. Starting from $b_{0}$ and given the actions and observations, we are then interested in checking whether the user can reach the high interest $\left(q_{3}\right)$ with a high certainty characterized by $b_{t}\left(q_{3}\right)$, ideally, $b_{t}\left(q_{3}\right)=1$. To this end, we calculate the reachable belief spaces using the sub-level sets of Lyapunov functions using optimization problem (34) and compare with simulations of the POMDP belief state evolutions. We fix the degree $d$ of variables $V_{a}, a=a_{0}, a_{1}$ and solve the optimization problem. The polynomial variable $p_{a}=\sum_{q \in\left\{q_{1}, q_{2}\right\}} b(q)^{d}$. Note that $b \in \mathcal{B}$, so $p_{a}$ is a positive polynomial.

Figure 7 illustrates the obtained results for local Lyapunov functions of degree one $d=1$ and degree three $d=3$ based on optimization problem (36). The calculations for the Lyapunov functions of degree 1 and degree 3 took 55.9480 and 203.4582 seconds, respectively. As it can be seen in the figure, simulations of the POMDP shows that the state $b\left(q_{3}\right)=1$ (i.e., $b\left(q_{1}\right)=b\left(q_{2}\right)=0$ ) is not reachable even with the policy $\pi$ due to the uncertainty of the problem and partial observation in the interest levels of the users. The over-approximation of the reachable belief space by local Lyapunov functions of degree one fails to capture this property; whereas, the overapproximation using local Lyapunov functions of degree three shows that the state $b\left(q_{1}\right)=b\left(q_{2}\right)=0$ is not reachable for the POMDP. Therefore, one can check reachability properties of POMDPs without the need for solving them explicitly by

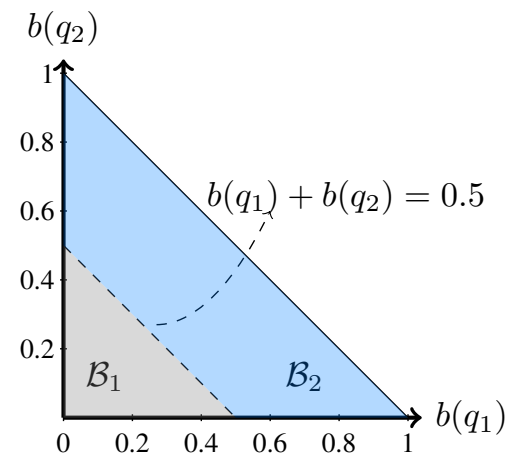

Fig. 6: The belief space in the interactive advertising example. The policy assigns actions $a_{0}$ (no ads) and $a_{1}$ (show ads) to different regions of the belief space $\mathcal{B}_{1}$ (high interest) and $\mathcal{B}_{2}$ (low to medium interest), respectively.
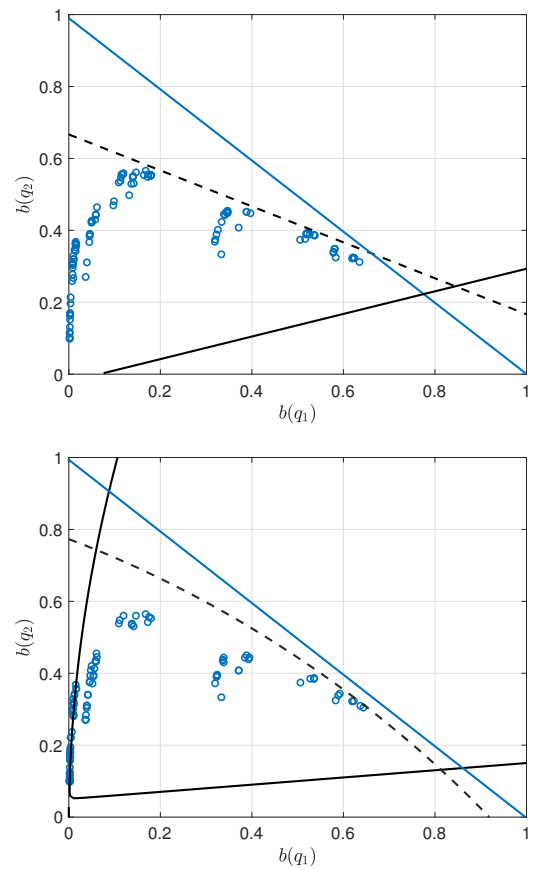

Fig. 7: The solutions of the POMDP (blue circles) in Case Study A and the approximated reachable belief space (contained between the black lines) for local Lyapunov functions of degree one (top) and degree three (below). The solid blue line demonstrates the boundary of the belief simplex.

considering a sufficiently large degree of the local Lyapunov functions.

\section{B. Machine Teaching}

In recent years, the need for machine learning systems has far exceeded the supply of machine learning experts. Machine teaching, that is, algorithms designed to enable machines to teach other machines or humans, have thus received attention [57]. Formally, machine teaching characterizes the algorithmic framework of designing an optimal training set for learning a target hypothesis [58]. The target hypothesis is given to the algorithm and the goal of the teacher (machine) is to generate a minimal sequence of training examples such 
that the target hypothesis can be learned by a learner (human or another machine) from a finite set of hypotheses.

We represent machine teaching as a sequential decision making under uncertainty scenario. To this end, we use a POMDP representation for the learner based on the statedependent teaching model (for more details see [59]). The POMDP model can be described as follows.

Definition 2 (Learning POMDP [59]). The learning POMDP $\mathcal{P}_{L}$ is a tuple $\left(Q, p_{0}, A, T, Z, O\right)$

- the hypotheses set $Q$ is a finite set of hidden states;

- $p_{0}$ is the probability of having an initial hypothesis $q_{0} \in$ $Q$;

- the set of examples A constitute the finite set of actions;

- the set of labels $Z$ constitute the finite set of observations;

- T describes the transitions from one hypothesis (state) to another characterized by a preference function;

- $O\left(z_{t} \mid q_{t}, a_{t}\right)$ determines the probability of seeing a label (observation) given the current hypothesis and example.

The learner starts with an initial hypothesis $q_{0}$, i.e., $b\left(q_{0}\right)=$ 1. Over a sequence of trials, in which an example $a_{t} \in A$ is shown and the learner receives a corresponding observation $z_{t} \in Z$, the learner updates its belief in the hypotheses.

We assume all hypotheses are uniformly distributed, or equivalently, $O\left(z_{t} \mid q_{t}, a_{t}\right)$ is binary. Moreover, the transition function $T\left(q, a_{t-1}, q^{\prime}\right)$ defines a uniform distribution: the learner only goes to the hypotheses $q^{\prime}$ that are the most preferred; hence, $T$ induces a uniform distribution over the most preferred hypothesis according to a preference function $\sigma$.

The learner starts with an initial hypothesis $q_{0}$ and over a sequence of trials, in which an example $a_{t} \in A$ is shown and the learner receives a corresponding observation $z_{t} \in Z$, develops a belief in the new hypothesis $q$.

In order to illustrate the proposed framework, we consider a toy scenario, where the teacher aims to teach/steer a human learner to reach a goal state in a physical environment. Each hypothesis/node corresponds to some unexplored territory, and there exists an example which flags the territory as explored. The learner prefers local moves, and if all neighboring territories are explored, the learner jumps to the next closest one.

The physical environment is characterized by a $4 \times 4$ lattice corresponding to 16 hypotheses. The teacher has 16 choices of locations on the lattice to show to the student as examples. The student then receives two labels based on its answer $y \in$ $\{-1,1\},-1$ for wrong and 1 for right. The preference function $\sigma\left(q^{\prime} ; q\right)$ is given by the minimum distance between hypotheses described by $\ell_{1}\left(q^{\prime} ; q\right)$.

In this example, we compare two teaching algorithms in the adaptive setting, where the teacher observes the learner's hypothesis at each iteration. The Myopic algorithm is a greedy approach which, at each iteration, picks the teaching example such that after observing the label, the worst-case rank of the target hypothesis in the learner's resulting version space is the smallest. The Ada-L algorithm aims to teach the learner some intermediate hypothesis at each iteration, i.e., it aims to direct the learner to transit to a hypothesis that is "closer" to the target hypothesis. For more details of the algorithms please refer to [53].
Each algorithm provides a set of policies for which we seek to find the minimum number of trials $\tau$ such that the belief in the target hypothesis $h^{*}$ is greater than a teaching performance $\lambda$

$$
b_{\tau}\left(q^{*}\right) \geq \lambda .
$$

To this end, we minimize the number of trials $\tau$ such that (43)-(45) are satisfied. We start by a large number of trials (16 in this case) and decrease it until no barrier certificate can be found to verify the teaching performance. We fix the degree of variables $B_{i}, p_{i}^{l_{1}}, p_{i}^{l_{2}}, p_{i}^{l_{3}}$, and $p_{i}^{f} \in \Sigma[b], i \in\{1,2, \ldots, N\}$ in Corollary 5 to 2 and search for the certificates. In order to check the SOS conditions, we use diagonally-dominantSOS (DSOS) relaxations of the SOS programs implemented through the SPOTless tool [60] (for more details see [61], [62]).

The results on finding the minimum number of trials $\tau$ for which the teaching performance is satisfied were as follows.

1) $q_{0}=(1,1)$ and $q^{*}=(3,3)$ : For the Myopic algorithm, for $\lambda=0.8$, we could find a certificate for $t=11$. Changing the teaching performance to $\lambda=0.6$ yielded certificates for only $\tau=9$. In contrast, for the Ada-L algorithm, we could obtain $\tau=6$ assuring teaching performance $\lambda=0.8$ and $\tau=$ 7 guaranteeing teaching performance $\lambda=0.9$. The calculation of the barrier certificates took on average 392.93 seconds with 0.05 increments of $\lambda$.

2) $q_{0}=(1,1)$ and $q^{*}=(3,4)$ : For the Myopic algorithm, we could not find any certificate for $\lambda=0.8$. Changing the the teaching performance to $\lambda=0.55$ yielded certificates for only $\tau=15$. On the other hand, for the Ada-L algorithm, we obtained $\tau=9$ assuring teaching performance $\lambda=0.8$ and $\tau=10$ assuring teaching performance $\lambda=0.9$. The calculation of the barrier certificates took on average 349.55 seconds with 0.05 increments of $\lambda$.

The results can also be corroborated from simulations. As can be see in Figures 8 and 9, the Myopic algorithm perform poorly on simple teaching tasks as compared to the Ada-L algorithm.

\section{CONCLUSIONS AND FUTURE WORK}

We studied the reachability, performance, and safety verification problems of POMDPs using methods from control theory, such as Lyapunov functions and barrier certificates. We showed how in several cases, our calculations can be decomposed and computationally implemented as a set of polynomial optimization problems. We used two examples to illustrate our proposed methodology.

Future work will include a synthesis algorithm for POMDPs. To this end, the literature on designing switching sequences of hybrid systems seems relevant [63]. Such synthesis techniques are aimed at providing guarantees for reachability, performance, and safety in POMDPs without explicitly or approximately solving them.

Our method was implemented as the solution to a set of sum-of-squares programs. The incorporation of special sparsity patterns in sum-of-squares programs has been shown to be very effective in reducing the computational burden of solving them. We will explore the possibility of using recent 

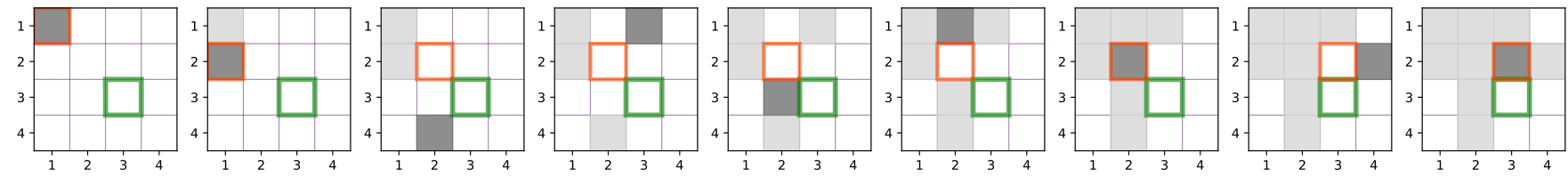

(a) Myopic
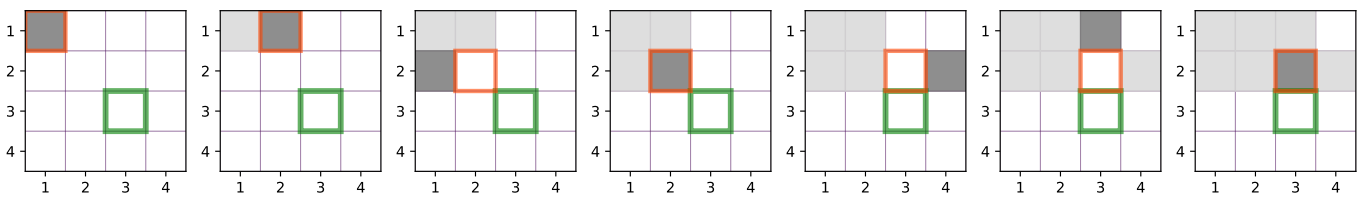

(b) Ada-L

Fig. 8: Teaching sequences generated by Myopic and Ada-L on a $4 \times 4$ lattice, with $q_{0}=(1,1), q^{*}=(3,3)$. The learner's current hypothesis is marked by orange, and the target is marked by green. The dark gray square represents the teaching example at the current time step, while light gray squares represent the previous teaching examples.
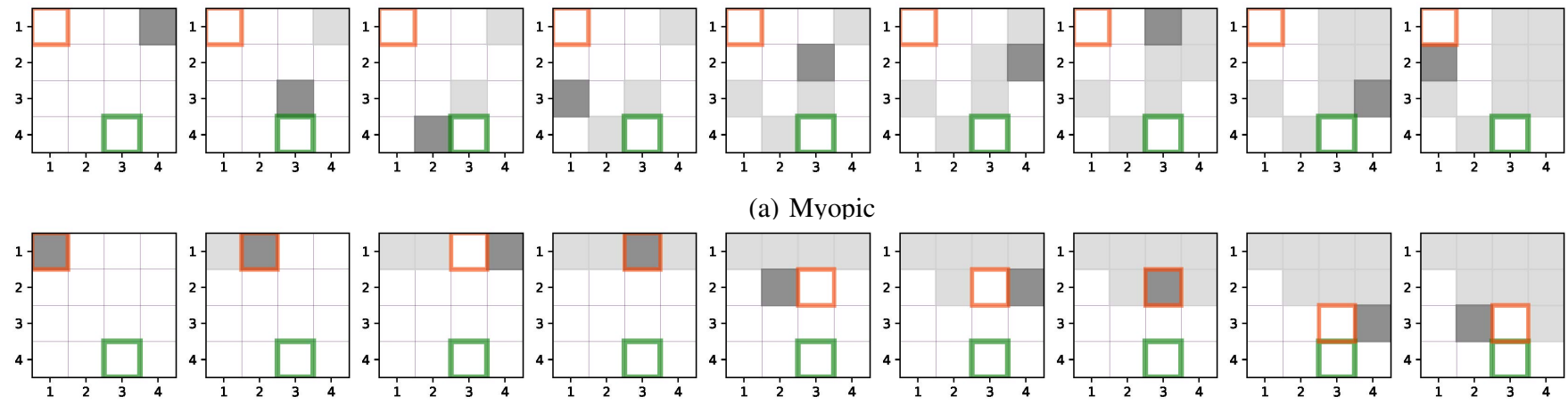

(a) Myopic
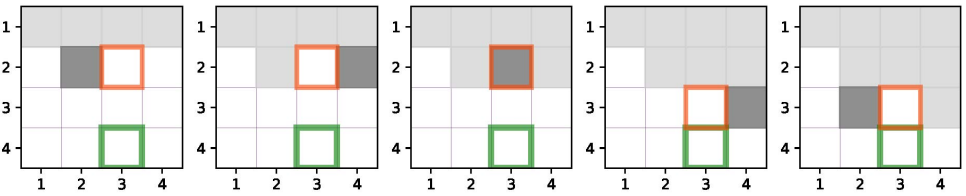

(b) Ada-L

Fig. 9: Teaching sequences generated by Myopic and Ada-L algorithms on a $4 \times 4$ lattice, with $q_{0}=(1,1), q^{*}=(3,4)$. The learner's initial hypothesis is marked by orange, and the target is marked by green. The dark gray square represents the teaching example at the current time step, while light gray squares represent the previous teaching examples.

results on chordal sparsity in sum-of-squares programs [64], [65] in our computational formulation. However, in general, even for systems described by polynomial/rational vector fields defined on semi-algebraic sets, the barrier certificates or the Lyapunov functions may not be polynomial. In two recent works [66], [67], one of the authors formulated discrete-time barrier functions to analyze POMDPs. Barrier functions are not restricted to polynomials and once the description of a safe set is given, the barrier function can be formulated in closed form [68]. Therefore, the method based on barrier functions can be used to circumvent the computational bottlenecks of sum-of-squares programming.

\section{ACKNOWLEDGMENTS}

M. Ahmadi appreciates the stimulating discussions with Dr. Yuxin Chen at the University of Chicago, Prof. Yisong Yue at Caltech, and Prof. Richard M. Murray at Caltech.

\section{APPENDIX}

\section{A. Sum-of-Squares Polynomials}

A polynomial $p(x)$ is a sum-of-squares polynomial if $\exists p_{i}(x) \in P[x], i \in\left\{1, \ldots, n_{d}\right\}$ such that $p(x)=\sum_{i} p_{i}^{2}(x)$.
Hence $p(x)$ is clearly non-negative. A set of polynomials $p_{i}$ is called SOS decomposition of $p(x)$. The converse does not hold in general, that is, there exist non-negative polynomials which do not have an SOS decomposition [69]. The computation of SOS decompositions, can be cast as an SDP (see [69], [70], [71]). The Theorem below proves that, in sets satisfying a property stronger than compactness, any positive polynomial can be expressed as a combination of sum-of-squares polynomials and polynomials describing the set.

For a set of polynomials $\bar{g}=\left\{g_{1}(x), \ldots, g_{m}(x)\right\}, m \in$ $\mathbb{Z}_{\geq 1}$, the quadratic module generated by $m$ is $M(\bar{g}):=$ $\left\{\sigma_{0}+\sum_{i=1}^{m} \sigma_{i} g_{i} \mid \sigma_{i} \in \Sigma[x]\right\}$. A quadratic module $M \in P[x]$ is said archimedean if $\exists N \in \mathbb{Z}_{\geq 1}$ such that $N-|x|^{2} \in M$. An Archimedian set is always compact [72]. At this point, we recall the following result [73, Theorem 2.14].

Theorem 7 (Putinar Positivstellensatz). Suppose the quadratic module $M(\bar{g})$ is Archimedian. Then for every $f \in P[x]$,

$$
f>0 \forall x \in\left\{x \mid g_{1}(x) \geq 0, \ldots, g_{m}(x) \geq 0\right\} \Rightarrow f \in(\bar{g}) .
$$

The subsequent proposition formalizes the problem of constrained positivity of polynomials which is a direct result of applying Positivstellensatz. 
Proposition 1 ([45]). Let $\left\{a_{i}\right\}_{i=1}^{k}$ and $\left\{b_{i}\right\}_{i=1}^{l}$ belong to $\mathcal{P}$, then

$$
\begin{gathered}
p(x) \geq 0 \quad \forall x \in \mathbb{R}^{n}: a_{i}(x)=0, \forall i=1,2, \ldots, k \\
\quad \text { and } \quad b_{j}(x) \geq 0, \forall j=1,2, \ldots, l
\end{gathered}
$$

is satisfied, if the following holds

$$
\begin{gathered}
\exists r_{1}, r_{2}, \ldots, r_{k} \in P[x] \text { and } \exists s_{0}, s_{1}, \ldots, s_{l} \in \Sigma[x] \\
p=\sum_{i=1}^{k} r_{i} a_{i}+\sum_{i=1}^{l} s_{i} b_{i}+s_{0}
\end{gathered}
$$

Proposition 2. The multivariable polynomial $p(x)$ is strictly positive $\left(p(x)>0 \quad \forall x \in \mathbb{R}^{n}\right)$, if there exists a $\lambda>0$ such that $(p(x)-\lambda) \in \Sigma[x]$.

\section{REFERENCES}

[1] M. L. Puterman, Markov Decision Processes: Discrete Stochastic Dynamic Programming. John Wiley and Sons, 1994.

[2] L. P. Kaelbling, M. L. Littman, and A. R. Cassandra, "Planning and acting in partially observable stochastic domains," Artificial Intelligence, vol. 101, no. 1, pp. 99-134, 1998.

[3] S. Thrun, W. Burgard, and D. Fox, Probabilistic Robotics. The MIT Press, 2005.

[4] T. Wongpiromsarn and E. Frazzoli, "Control of probabilistic systems under dynamic, partially known environments with temporal logic specifications," in CDC. IEEE, 2012, pp. 7644-7651.

[5] S. J. Russell and P. Norvig, Artificial Intelligence: A Modern Approach, 2nd ed. Pearson Education, 2003.

[6] G. Shani, J. Pineau, and R. Kaplow, "A survey of point-based POMDP solvers," Autonomous Agents and Multi-Agent Systems, vol. 27, no. 1, pp. 1-51, 2013.

[7] O. Madani, S. Hanks, and A. Condon, "On the undecidability of probabilistic planning and infinite-horizon partially observable Markov decision problems," in AAAI. AAAI Press, 1999, pp. 541-548.

[8] D. Braziunas, "Pomdp solution methods," University of Toronto, Tech. Rep., 2003.

[9] K. Chatterjee, M. Chmelík, and M. Tracol, "What is decidable about partially observable Markov decision processes with $\omega$-regular objectives," Journal of Computer and System Sciences, vol. 82, no. 5, pp. 878-911, 2016.

[10] M. Hauskrecht, "Value-function approximations for partially observable markov decision processes," J. Artif. Int. Res., vol. 13, no. 1, pp. 33-94, Aug. 2000.

[11] B. Wu and $\mathrm{H}$. Lin, "Privacy verification and enforcement via belief abstraction," IEEE control systems letters, vol. 2, no. 4, pp. 815-820, 2018.

[12] M. T. J. Spaan and N. Vlassis, "Perseus: Randomized point-based value iteration for POMDPs," J. Artif. Int. Res., vol. 24, no. 1, pp. 195-220, Aug. 2005.

[13] O. Brock, J. Trinkle, and F. Ramos, SARSOP: Efficient Point-Based POMDP Planning by Approximating Optimally Reachable Belief Spaces. MIT Press, 2009, pp. 65-72.

[14] D. Hsu, W. S. Lee, and N. Rong, "Accelerating point-based POMDP algorithms through successive approximations of the optimal reachable space," 2007.

[15] J. Pineau, G. Gordon, and S. Thrun, "Anytime point-based approximations for large POMDPs," Journal of Artificial Intelligence Research, vol. 27, pp. 335-380, 2006.

[16] W. S. Lee, N. Rong, and D. Hsu, "What makes some POMDP problems easy to approximate?" in Advances in neural information processing systems, 2008, pp. 689-696.

[17] H. Kurniawati, D. Hsu, and W. S. Lee, "SARSOP: Efficient point-based POMDP planning by approximating optimally reachable belief spaces." in Robotics: Science and systems, vol. 2008. Zurich, Switzerland., 2008.

[18] M. Spaan and N. Vlassis, "Perseus: Randomized point-based value iteration for POMDPs," Journal of artificial intelligence research, vol. 24, pp. 195-220, 2005.

[19] Y. Luo, H. Bai, D. Hsu, and W. S. Lee, "Importance sampling for online planning under uncertainty," The International Journal of Robotics Research, p. 0278364918780322, 2016.
[20] A. Agha-Mohammadi, S. Agarwal, A. Mahadevan, S. Chakravorty, D. Tomkins, J. Denny, and N. M. Amato, "Robust online belief space planning in changing environments: Application to physical mobile robots." in ICRA, 2014, pp. 149-156.

[21] S. Prentice and N. Roy, "The belief roadmap: Efficient planning in linear POMDPs by factoring the covariance," in Robotics Research. Springer, 2010, pp. 293-305.

[22] M. J. Kochenderfer and J. P. Chryssanthacopoulos, "Collision avoidance using partially controlled Markov decision processes," in Agents and Artificial Intelligence, J. Filipe and A. Fred, Eds. Springer, 2013, vol. 271 , pp. 86-100.

[23] T. Smith and R. Simmons, "Heuristic search value iteration for POMDPs," in Proceedings of the 20th conference on Uncertainty in artificial intelligence. AUAI Press, 2004, pp. 520-527.

[24] M. Ahmadi, B. Wu, H. Lin, and U. Topcu, "Privacy verification in POMDPs via barrier certificates," in Decision and Control (CDC), 2018 IEEE 57th Annual Conference on, 2018.

[25] M. Ahmadi, M. Cubuktepe, N. Jansen, and U. Topcu, "Verification of uncertain POMDPs using barrier certificates," in 56th Annual Allerton Conference on Communication, Control, and Computing,, 2018.

[26] E. J. Sondik, "The optimal control of partially observable Markov processes over the infinite horizon: Discounted costs," Operations Research, vol. 26, no. 2, pp. 282-304, 1978.

[27] K. J. Aström, "Optimal control of Markov decision processes with incomplete state estimation," J. Math. Anal. Appl., vol. 10, pp. 174$205,1965$.

[28] R. E. Kalman, "A new approach to linear filtering and prediction problems," Journal of basic Engineering, vol. 82, no. 1, pp. 35-45, 1960.

[29] R. Goebel, R. G. Sanfelice, and A. R. Teel, "Hybrid dynamical systems," IEEE Control Systems, vol. 29, no. 2, pp. 28-93, 2009.

[30] A. A. Ahmadi and P. A. Parrilo, "Non-monotonic Lyapunov functions for stability of discrete time nonlinear and switched systems," in Decision and Control, 2008. CDC 2008. 47th IEEE Conference on. IEEE, 2008, pp. 614-621.

[31] A. Kundu and D. Chatterjee, "On stability of discrete-time switched systems," Nonlinear Analysis: Hybrid Systems, vol. 23, pp. 191 - 210, 2017.

[32] W. Zhang, A. Abate, J. Hu, and M. P. Vitus, "Exponential stabilization of discrete-time switched linear systems," Automatica, vol. 45, no. 11, pp. 2526-2536, 2009

[33] M. Hauskrecht, "Value-function approximations for partially observable Markov decision processes," Journal of artificial intelligence research, vol. 13, pp. 33-94, 2000

[34] D. Liberzon, Switching in Systems and Control, ser. Systems \& Control: Foundations \& Applications. Birkhäuser Boston, 2003.

[35] J. P. Hespanha, "Uniform stability of switched linear systems: Extensions of LaSalle's invariance principle," IEEE Transactions on Automatic Control, vol. 49, no. 4, pp. 470-482, 2004.

[36] M. Steinmetz, J. Hoffmann, and O. Buffet, "Goal probability analysis in probabilistic planning: Exploring and enhancing the state of the art," J. Artif. Intell. Res., vol. 57, pp. 229-271, 2016.

[37] A. Kolobov, "Planning with Markov decision processes: An AI perspective," Synthesis Lectures on Artificial Intelligence and Machine Learning, vol. 6, no. 1, pp. 1-210, 2012.

[38] J.-P. Katoen, "The probabilistic model checking landscape," in LICS. ACM, 2016, pp. 31-45.

[39] F. Blanchini and S. Miani, Set-theoretic methods in control. Springer, 2008.

[40] F. Berkenkamp, R. Moriconi, A. P. Schoellig, and A. Krause, "Safe learning of regions of attraction for uncertain, nonlinear systems with gaussian processes," in Decision and Control (CDC), 2016 IEEE 55th Conference on. IEEE, 2016, pp. 4661-4666.

[41] B. Legat, R. Jungers, and P. Parrilo, "Computing controlled invariant sets for hybrid systems with applications to model-predictive control," arXiv preprint arXiv:1802.04522, 2018.

[42] H. Burchardt and S. Ratschan, "Estimating the region of attraction of ordinary differential equations by quantified constraint solving," in Proceedings Of The 3rd WSEAS International Conference On Dynamical Systems And Control, vol. 241. Citeseer, 2007.

[43] T. Gurriet, A. Singletary, J. Reher, L. Ciarletta, E. Feron, and A. Ames, "Towards a framework for realizable safety critical control through active set invariance," in Proceedings of the 9th ACM/IEEE International Conference on Cyber-Physical Systems, ser. ICCPS '18. Piscataway, NJ, USA: IEEE Press, 2018, pp. 98-106.

[44] A. Packard, U. Topcu, P. Seiler, and G. Balas, "Help on sos," IEEE Control Systems, vol. 30, no. 4, pp. 18-23, 2010. 
[45] G. Chesi, "LMI techniques for optimization over polynomials in control: a survey," IEEE Transactions on Automatic Control, vol. 55, no. 11, pp. $2500-2510,2010$

[46] A. Milias-Argeitis and M. Khammash, "Optimization-based lyapunov function construction for continuous-time markov chains with affine transition rates," in 53rd IEEE Conference on Decision and Control. IEEE, 2014, pp. 4617-4622.

[47] M. Ahmadi, M. Cubuktepe, N. Jansen, and U. Topcu, "Verification of uncertain pomdps using barrier certificates," in 2018 56th Annual Allerton Conference on Communication, Control, and Computing (Allerton). IEEE, 2018, pp. 115-122.

[48] M. Ahmadi, G. Valmorbida, and A. Papachristodoulou, "Barrier functionals for output functional estimation of PDEs," in 2015 American Control Conference (ACC), 2015, pp. 2594-2599.

[49] _ _ "Safety verification for distributed parameter systems using barrier functionals," Systems \& Control Letters, vol. 108, pp. 33 - 39, 2017.

[50] M. Ahmadi, A. Israel, and U. Topcu, "Safety assessment based on physically-viable data-driven models," in 2017 IEEE 56th Annual Conference on Decision and Control (CDC), Dec 2017, pp. 6409-6414.

[51] M. Ahmadi, A. Israel, and U. Topcu, "Safe Controller Synthesis for Data-Driven Differential Inclusions," IEEE Transactions on Automatic Control, 2019.

[52] M. H. Stone, "Applications of the theory of Boolean rings to general topology," Transactions of the American Mathematical Society, vol. 41, no. 3, pp. 375-481, 1937.

53] Y. Chen, A. Singla, O. M. Aodha, P. Perona, and Y. Yue, "Understanding the role of adaptivity in machine teaching: The case of version space learners," in Proc. Conference on Neural Information Processing Systems (NIPS), December 2018.

[54] V. Krishnamurthy, A. Aprem, and S. Bhatt, "Multiple stopping time pomdps: Structural results \& application in interactive advertising on social media," Automatica, vol. 95, pp. 385-398, 2018.

[55] K. Yadati, H. Katti, and M. Kankanhalli, "Cavva: Computational affective video-in-video advertising," IEEE Transactions on Multimedia, vol. 16 , no. 1 , pp. 15-23, 2014

[56] J. Lehmann, M. Lalmas, E. Yom-Tov, and G. Dupret, "Models of user engagement," in International Conference on User Modeling, Adaptation, and Personalization. Springer, 2012, pp. 164-175.

[57] P. Y. Simard, S. Amershi, D. M. Chickering, A. E. Pelton, S. Ghorashi, C. Meek, G. Ramos, J. Suh, J. Verwey, M. Wang et al., "Machine teaching: A new paradigm for building machine learning systems," arXiv preprint arXiv:1707.06742, 2017.

[58] X. Zhu, "Machine teaching: An inverse problem to machine learning and an approach toward optimal education." in AAAI, 2015, pp. 4083-4087.

[59] M. Ahmadi, B. Wu, Y. Chen, Y. Yue, and U. Topcu, "Barrier certificates for assured machine teaching," in 2019 American Control Conference, 2019.

[60] A. Megretski, "Systems polynomial optimization tools (SPOT)," 2010. [Online]. Available: https://github.com/anirudhamajumdar/spotless/tree/spotless_isos

[61] A. A. Ahmadi and A. Majumdar, "DSOS and SDSOS optimization: more tractable alternatives to sum of squares and semidefinite optimization," arXiv preprint arXiv:1706.02586, 2017.

[62] A. A. Ahmadi, G. Hall, A. Papachristodoulou, J. Saunderson, and Y. Zheng, "Improving efficiency and scalability of sum of squares optimization: Recent advances and limitations," in 2017 IEEE 56th Annual Conference on Decision and Control (CDC), Dec 2017, pp. 453462.

[63] B. Stellato, S. Ober-Blöbaum, and P. J. Goulart, "Second-order switching time optimization for switched dynamical systems," IEEE Transactions on Automatic Control, vol. 62, no. 10, pp. 5407-5414, 2017.

[64] Y. Zheng, G. Fantuzzi, and A. Papachristodoulou, "Sparse sum-ofsquares (SOS) optimization: A bridge between DSOS/SDSOS and SOS optimization for sparse polynomials," arXiv preprint arXiv:1807.05463, 2018.

[65] A. A. Ahmadi, G. Hall, A. Papachristodoulou, J. Saunderson, and Y. Zheng, "Improving efficiency and scalability of sum of squares optimization: Recent advances and limitations," in Decision and Control (CDC), 2017 IEEE 56th Annual Conference on. IEEE, 2017, pp. 453462.

[66] M. Ahmadi, J. W. Singletary, A.and Burdick, and A. D. Ames, "Safe policy synthesis in multi-agent POMDPs via discrete-time barrier functions," in 58th IEEE Conference on Decision and Control, 2019.

[67] M. Ahmadi, A. Singletary, J. W. Burdick, and A. D. Ames, "Barrier Functions for Multiagent-POMDPs with DTL Specifications," arXiv preprint arXiv:2003.09267, 2020
[68] A. D. Ames, X. Xu, J. W. Grizzle, and P. Tabuada, "Control barrier function based quadratic programs for safety critical systems," IEEE Transactions on Automatic Control, 2016.

[69] P. Parrilo, "Structured semidefinite programs and semialgebraic geometry methods in robustness and optimization," Ph.D. dissertation, California Institute of Technology, 2000

[70] M. Choi, T. Y. Lam, and B. Reznick, "Sums of squares of rea polynomials," in Proceedings of Symposia in Pure mathematics, vol. 58. American Mathematical Society, 1995, pp. 103-126.

[71] G. Chesi, A. Tesi, A. Vicino, and R. Genesio, "On convexification of some minimum distance problems," in 5th European Control Conference, Karlsruhe, Germany, 1999.

[72] M. Nie, J.and Schweighofer, "On the complexity of Putinar's positivstellensatz," Journal of Complexity, vol. 23, no. 1, pp. 135-150, 2007.

[73] J. B. Lasserre, Moments, Positive Polynomials and Their Applications. Imperial College Press, London, 2009.

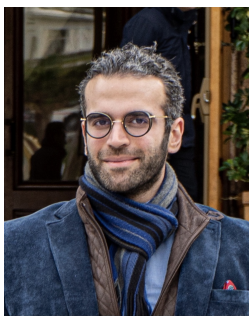

Mohamadreza Ahmadi is a senior postdoctora scholar at the Center for Autonomous Systems and Technologies (CAST) at the California Institute of Technology, CA. He finished his DPhil in Engineering Science (Control Systems and Aeronautics) in November 2016 at the University of Oxford, UK, as a Clarendon Scholar. His PhD was followed by research positions at the University of Texas at Austin. His current research is on multi-agent decision making under uncertainty with application to artificial intelligence, space, and robotics systems

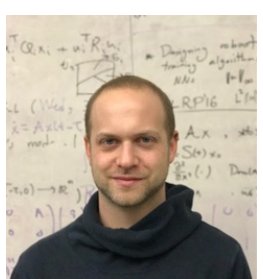

Nils Jansen is an assistant professor at the Institute for Computing and Information Science (iCIS) at the Radboud University, Nijmegen, The Netherlands. He received his Ph.D. in computer science from RWTH Aachen University, Germany in 2015. Prior to Radboud University, he was a postdoc and research associate at the University of Texas at Austin His current research is on formal reasoning about safety aspects in machine learning. At the heart is the development of concepts from formal methods to reason about uncertainty and partial observability.

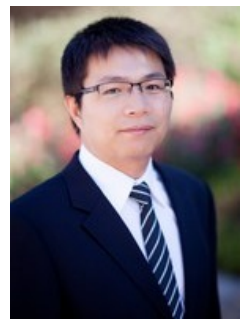

Bo Wu received the B.S. degree from Harbin Institute of Technology, China, in 2008, M.S. degree from Lund University, Sweden, in 2011 and a Ph.D degree from University of Notre Dame, USA, in 2018, all in electrical engineering. He is currently a postdoctoral researcher in the Oden Institute for Computational Engineering and Sciences at the University of Texas at Austin. His research interest is to apply formal methods, learning, and optimization in autonomous systems, such as robotic systems, communication systems, and human-in-the-loop systems, to provide security and performance guarantees.

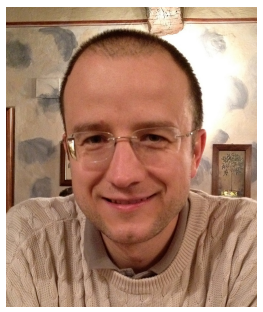

Ufuk Topcu joined the Department of Aerospace Engineering at the University of Texas at Austin as an assistant professor in Fall 2015. He received his Ph.D. degree from the University of California at Berkeley in 2008. He held research positions at the University of Pennsylvania and California Institute of Technology. His research focuses on the theoretical, algorithmic and computational aspects of design and verification of autonomous systems through novel connections between formal methods, learning theory and controls. 